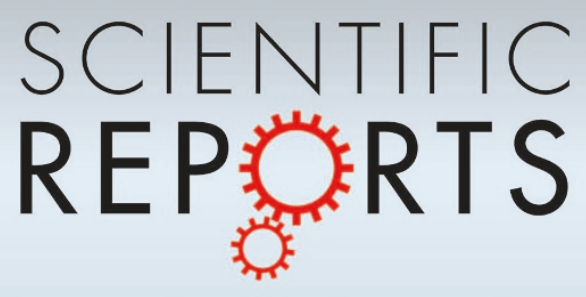

OPEN

SUBJECT AREAS:

STEM-CELL

BIOTECHNOLOGY

BIOMEDICAL ENGINEERING

Received

4 February 2014

Accepted

24 March 2014

Published

10 April 2014

Correspondence and requests for materials should be addressed to

J.M.K. (jmkarp@ partners.org)

\section{Performance-enhanced mesenchymal stem cells via intracellular delivery of steroids}

\author{
James A. Ankrum ${ }^{1,2}$, Riddhi G. Dastidar', Joon Faii Ong', Oren Levy' \& Jeffrey M. Karp ${ }^{1,2,3}$
}

\begin{abstract}
'Center for Regenerative Therapeutics, Department of Medicine, Brigham and Women's Hospital, Harvard Medical School, Boston, MA 021 15, USA, ${ }^{2}$ Harvard-MIT Division of Health Sciences \& Technology, Cambridge, MA 02139, USA, ${ }^{3}$ Harvard Stem Cell Institute, 1350 Massachusetts Avenue, Cambridge, MA 02138, USA.
\end{abstract}

Inadequate immunomodulatory potency of mesenchymal stem cells (MSC) may limit their therapeutic efficacy. We report glucocorticoid steroids augment MSC expression and activity of indoleamine-2,3-dioxygenase (IDO), a primary mediator of MSC immunomodulatory function. This effect depends on signaling through the glucocorticoid receptor and is mediated through up-regulation of FOXO3. Treatment of MSCs with glucocorticoids, budesonide or dexamethasone, enhanced IDO expression following IFN- $\gamma$ stimulation in multiple donors and was able to restore IDO expression in over-passaged MSCs. As IDO enhancement was most notable when cells were continuously exposed to budesonide, we engineered MSC with budesonide loaded PLGA microparticles. MSC efficiently internalized budesonide microparticles and exhibited 4-fold enhanced IDO activity compared to budesonide preconditioned and naive MSC, resulting in a 2-fold improvement in suppression of stimulated peripheral blood mononuclear cells in an IDO-dependent manner. Thus, the augmentation of MSC immune modulation may abrogate challenges associated with inadequate potency and enhance their therapeutic efficacy.

T

he potential of mesenchymal stem cells (MSCs) to ameliorate inflammation arising from numerous diseases has been established in preclinical animal models leading to hundreds of MSC clinical trials ${ }^{1,48}$. While MSCs appear to be beneficial in several disease models, treatment of patients has led to highly variable outcomes. For example, subsets of patients with graft versus host disease (GvHD) have responded remarkably well to MSC therapy with at least temporary resolution of symptoms ${ }^{2,3}$, while for others their prognosis is unaltered ${ }^{4,5}$. Factors limiting MSC therapy include insufficient and variable immunomodulatory potency ${ }^{6-8}$, dependence on in vivo activation by host inflammatory mediators to achieve MSC immunomodulation ${ }^{7-9}$, and limited MSC persistence $^{10,11}$. Substantial batch to batch variability in MSC secretome ${ }^{8}$, in vitro immunomodulatory potential ${ }^{1,6,7}$, and treatment efficacy linked to differences in MSC donors ${ }^{2,3,6,7}$, tissue of origin ${ }^{4-6,12}$, and passages ${ }^{2,6-8}$ have recently been reported. Furthermore, limited persistence shortens the therapeutic window in which MSCs modulate inflammatory responses via secretion of growth factors and cytokines, release of exosomes, or activity of immunomodulatory enzymes such as iNOS and IDO. Thus, techniques to augment and control the potency of MSC are needed for a therapeutic effect to be exerted within a short therapeutic window. We hypothesize that engineering MSCs to maximize and sustain immunosuppressive potential will enable the generation of enhanced cell-based therapies and eliminate the need to contemplate from which tissue or donor MSCs should be derived.

One of the primary factors mediating MSC immune suppression is the tryptophan depleting enzyme indoleamine-2,3-dioxygenase (IDO). Inhibiting IDO with 1-methyl-DL-tryptophan (1-MT) in human MSCs abrogates their immunosuppressive potential in peripheral blood mononuclear cell (PBMC) co-cultures ${ }^{7-9,13}$. In contrast to secreted factors implicated in MSC immune suppression such as PGE2 $2^{10,11,14}$ or TSG-6 $6^{8,10}$, IDO is an intracellular enzyme. Specifically, IDO is the first and rate-limiting enzyme involved in degradation of tryptophan down the kynurenine pathway and is predominately expressed in antigen presenting cells (APCs) in response to type I interfons ${ }^{15}$. Additionally, human MSCs have been shown to have IDO-dependent antimicrobial affects against staphylococcus aureus, staphylococcus epidermis, and toxoplasma gondii ${ }^{16}$. As in APCs, expression of IDO in MSCs occurs in response to inflammation, induced by exposure to interferon- $\gamma$ (IFN- $\gamma$ ). Both the depletion of tryptophan and the generation of kynurenine byproducts have potent suppressive effects on immune cells ${ }^{15,17}$. IDO has been implicated in promoting both physiological and pathological tolerance. IDO expressed by cells in the placenta is responsible for fetal tolerance, and inhibition of IDO by the inhibitor 
1-methyl-tryptophan (1-MT) results in allograft rejection of the fetus ${ }^{18}$. More recently, IDO has been found to be overexpressed in solid tumors, promoting tolerance towards tumor antigens ${ }^{19}$ and tryptophan depletion has been shown to suppress T-cell proliferation through activation of general control non-depressing 2 (GCN2) kinase $^{20}$. In addition to tryptophan depletion, IDO generates tryptophan catabolites that inhibit the proliferation of activated T-cells ${ }^{21}$ and induce naïve T-cells to become FoxP3 + T-regulatory cells ${ }^{22}$. Due to the role of IDO as a negative regulator of inflammation and inducer of tolerance, the level and regulation of its expression is of great interest for maternal-fetal tolerance, tumor immunity, allergy, autoimmune disease, transplant tolerance, and MSC therapy. Unfortunately, the level of MSC IDO expression varies significantly between donors and between MSC tissue sources leading to variability in MSC's ability to suppress activated T-cells ${ }^{6,7}$.

As elevated IDO activity correlates with enhanced suppression of $\mathrm{T}$-cell activation and proliferation, augmenting MSC IDO levels should increase their immunomodulatory potency. Interestingly, IDO expression in macrophages and dendritic cells has been shown to be augmented by exposure to glucocorticoid steroids mediated in part through cell-cell contact signaling through interactions between the glucocorticoid-induced tumor necrosis factor receptor related protein (GITR) and its ligand (GITRL) ${ }^{23,24}$. Similarly, augmented IDO expression following glucocorticoid steroid treatment has also been observed in astrocytes, although the mechanism of steroid enhanced IDO activity is not known ${ }^{25}$. While MSCs are frequently administered to patients with GvHD who are already receiving glucocorticoid steroids $s^{3,26}$, the impact of glucocorticoid steroids on MSCs, and specifically on their IDO activity, has not been investigated.

Herein, we report that budesonide, a glucocorticoid steroid with fewer systemic effects than dexamethasone ${ }^{27}$, significantly boosts MSC IDO activity and immunosuppressive phenotype and we introduce a cell engineering strategy to sustain MSC therapeutic potency without requiring soluble budesonide. Specifically, we observe that budesonide-treated MSCs show similar metabolic activity, viability, and morphology to untreated MSCs, yet exhibit a favorable shift in their expression of immunogenic and immunosuppressive factors. In addition to enhancing IFN- $\gamma$ induced IDO expression and activity, BUD treatment also significantly reduced MSC expression of MHC molecules. We found that combined exposure to both budesonide and inflammatory cues resulted in the highest levels of IDO expression, as IDO is only expressed in response to inflammatory signaling. Additionally, the response was broadly applicable to other glucocorticoids, as dexamethasone produced a similar IDO response, and blocking the glucocorticoid receptor inhibited the enhanced IDO expression. To engineer cells with enhanced and sustained immunosuppressive potency that can be activated upon entering an inflammatory environment without requiring systemic administration of immunosuppressant drugs, we utilized a particle engineering approach. Budesonide loaded microparticles were efficiently internalized by MSCs and resulted in significant enhancement of MSC IDO expression and activity, similar to soluble budesonide. In vitro co-culture assays with PBMCs revealed the engineered MSCs could be activated in situ in response to IFN- $\gamma$ produced by PBMCs. Engineered MSC suppression of PBMCs was enhanced further by pre-activating MSCs with IFN- $\gamma$ to increase expression of IDO prior to the initiation of the PBMC co-cultures. Addition of the IDO inhibitor 1-MT completely abolished the suppressive properties of the engineered MSCs, implicating IDO as a key immunosuppressive factor. We believe this engineering strategy could be used to augment MSC potency, resulting in enhanced cell-based therapies.

\section{Results}

Impact of budesonide on MSC viability and morphology. Glucocorticoid steroids are commonly prescribed anti-inflammatory drugs that have diverse effects on immune cells, however their effect on MSC immunogenicity and immunomodulatory potential has not been thoroughly examined. Glucocorticoid steroids exert their effects through binding cytoplasmic glucocorticoid receptors and translocating to the nucleus where they bind glucocorticoid responive elements (GREs), resulting in promotion or suppression of gene expression $^{28}$. As such, glucocorticoid steroids, such as budesonide, have variable effects on cells of different origins, including growth inhibition $^{29}$, suppression of MHC expression ${ }^{28,30}$, and induction of $\mathrm{IDO}^{25}$.

To examine if budesonide alters MSC potency without negatively impacting MSC phenotype, MSCs were exposed to 0.001-100 $\mu \mathrm{M}$ budesonide for 24-72 hours. Metabolic activity, as assessed by XTT, was not significantly affected by budesonide treatment for 24,48 , or 72 hours over the dosage range tested (Fig. 1A). Flow cytometry analysis revealed no significant changes in MSC viability as evidenced by dual negative staining for Annexin- $\mathrm{V}$ and propidium iodide after 72 hours of budesonide exposure (Fig. 1B). Phase contrast imaging of treated cells did not show any appreciable impact of budesonide on the morphology of MSCs after 48 hours of budesonide exposure (Fig. 1C). In addition, MSCs treated with budesonide for 48 hours showed no evidence of increased cell death when evaluated for double stranded DNA breaks by TUNEL staining (Fig. 1D). Collectively these data show MSC metabolic activity, morphology, proliferation, and viability were preserved over a wide range of budesonide concentrations.

Impact of budesonide on MSC immunophenotype. Next we analyzed the effect of budesonide on MSC expression of MHC I and MHC II molecules. The naturally low expression of MHC I, lack of MHC II, and failure of MSC to elicit hyperacute rejection upon infusion has been used to justify the use of unmatched allogeneic MSCs in animal models and clinical trials. However, upon exposure to an inflammatory environment, expression of both MHC molecules increases ${ }^{31}$. Interestingly, glucocorticoids have been shown to reduce expression of MHC molecules in many cell type ${ }^{28,30}$. Expression of class I and class II MHC molecules, HLA$A B C$ and HLA-DR respectively, were examined to investigate budesonide's impact on MSC immunophenotype before and after IFN- $\gamma$ stimulation. MSCs were exposed to vehicle (DMSO) or budesonide for 24 hours after which media was changed to include IFN- $\gamma$ and the cells were cultured for an additional 48 hours prior to antibody staining and flow cytometry analysis. Cells in the 'drug preconditioning' group were exposed to budesonide only during the first 24 hours while cells in the 'drug sustained' group were exposed to budesonide for the duration of the experiment (Fig. 2A). Figure 2B shows that MSC expression of HLA-ABC was reduced by over $50 \%$ for all doses of budesonide in both the precondition and drug sustained groups $(\mathrm{p}<0.001)$. Following exposure to $100 \mu \mathrm{M}$ budesonide, stimulation with IFN- $\gamma$ reduced HLA-ABC expression by only $\sim 15 \%$ (not significant (NS), Fig. $2 B$, C). Budesonide treatment did not result in any increase of HLA-DR expression in unstimulated conditions or decrease in HLA-DR in stimulated conditions except for the $100 \mu \mathrm{M}$ treated group. Preconditioning with $100 \mu \mathrm{M}$ budesonide reduced IFN- $\gamma$ induced HLA-DR expression by $11 \%$ (NS) while sustained exposure resulted in a $40 \%$ reduction ( $p<0.001$, Fig. $2 D, E)$.

Impact of budesonide on MSC IDO activity. MSC immunosuppressive potential was evaluated following exposure to budesonide through assessment of IDO protein content and activity. Following treatment of MSCs with $1 \mu \mathrm{M}$ budesonide for 24 hours, MSCs were additionally exposed to $100 \mathrm{ng} / \mathrm{ml} \mathrm{IFN-} \gamma$ for 48 hours (Fig. 3A). MSCs were collected, lysed, and IDO protein content was analyzed by western blot. Budesonide treatment alone did not result in any increase in IDO protein content over untreated MSCs (Fig. 3B). IFN$\gamma$ stimulation resulted in increased IDO content in both untreated 
A

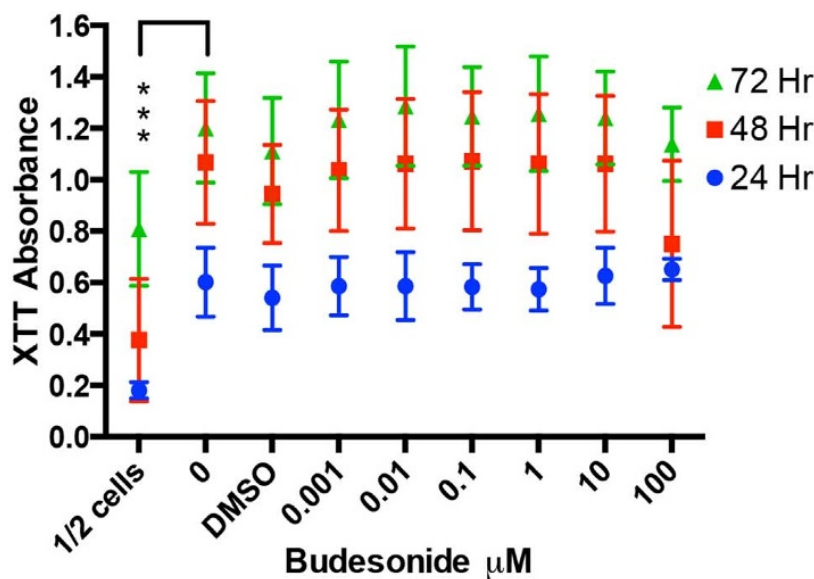

B

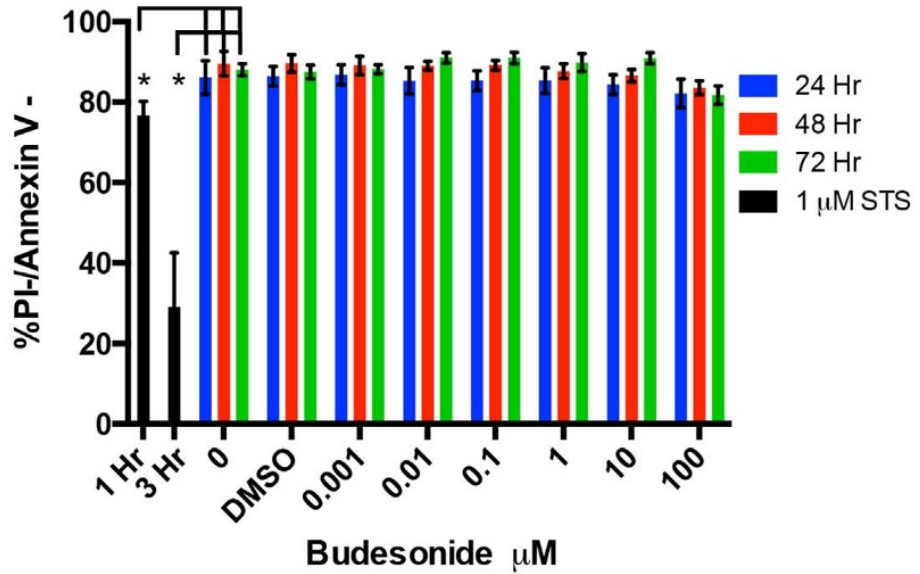

$\mathrm{D}$
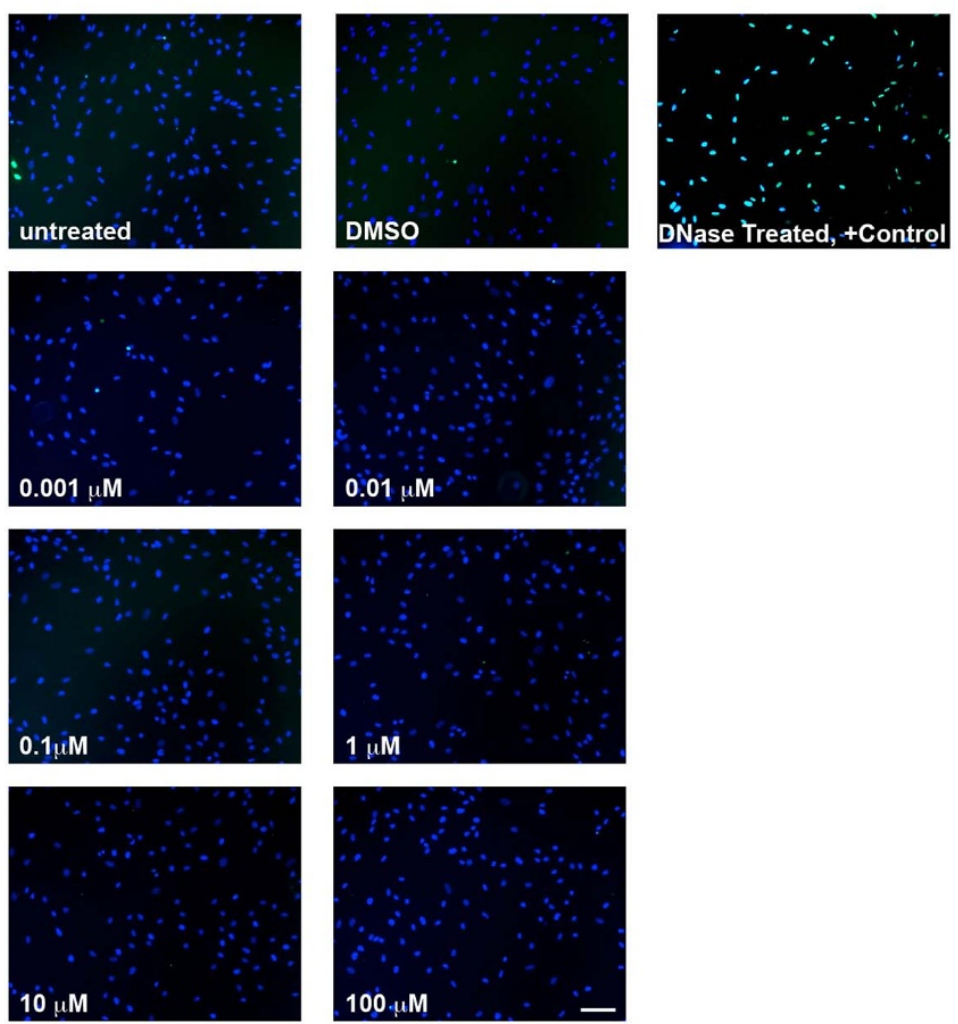

Figure 1 Viability, metabolic activity, and morphology of budesonide treated MSC (A) MSC were plated in 96 well plates (15,000 cells/well) treated with DMSO or 0.001-100 uM budesonide and their metabolic activity was assessed by XTT at 24,48 , and 72 hours. $1 / 2$ cell condition (7,500 cells/ well) used as control to show a reduction in XTT signal when fewer cells are present. (B) MSCs were plated in 24 well plates (30,000 cells/well) treated with DMSO or 0.001-100 uM budesonide. At 24, 48, and 72 hours after plating, cells were harvested, and viability was measured by staining with Annexin V and propidium iodide and analyzing by flow cytometry. Staurosporine (STS) treatment for 1 and 3 hour was used as a positive control for cell death. (C) Representative phase contrast images of MSCs treated with budesonide for 48 hours show no change in morphology, density, or adherence.

(D) TUNEL staining for double stranded DNA breaks after 48 hour budesonide exposure shows no increase in apoptosis. Stain control cells were treated with DNase to induce DNA breaks and served as a positive control for TUNEL stain. (DAPI, Blue; TUNEL, Green). (Bars are mean \pm SEM, 2-way ANOVA with Fishers LSD test, $\left.\mathrm{n}=3,{ }^{*} \mathrm{p}<0.05\right)$ Scale bar $100 \mu \mathrm{m}$.

and budesonide treated MSCs, with budesonide treated cells containing significantly more IDO than untreated controls. To determine the extent to which budesonide could enhance MSC IDO expression, IDO content of MSCs from multiple donors and passages was analyzed with and without budesonide treatment. Enhancement in IDO expression following budesonide exposure was observed in MSCs from multiple donors, regardless of the baseline level of IDO expression (no budesonide treatment) for each donor (Fig. 3C). In addition, high passage, P8, MSCs treated with budesonide expressed IDO at levels greater or similar to untreated P5 MSCs (Fig. 3D). Interestingly, IDO expression was most significantly enhanced when budesonide was continuously present versus simply pretreated (Fig. 3E,F). To demonstrate the ability to enhance the immunomodulatory potency of MSCs with poor immunosuppressive potential, donor \#7083, which had the lowest baseline IFN- $\gamma$ inducible IDO expression (no budesonide 


\section{A Drug Precondition}

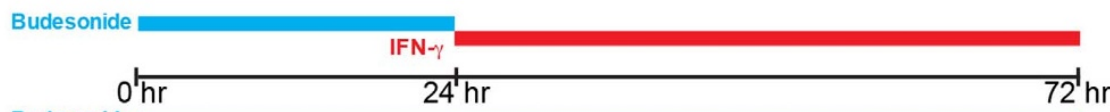

Drug Sustained

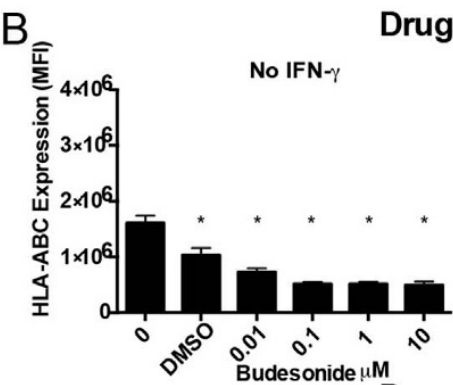

Drug Precondition
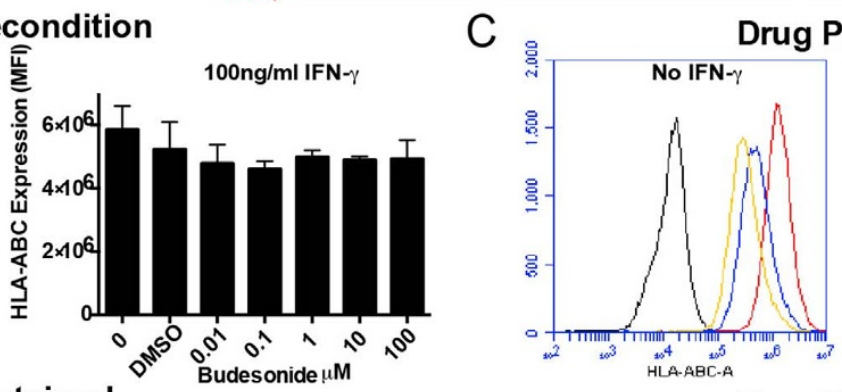

Drug Precondition

Drug Sustained
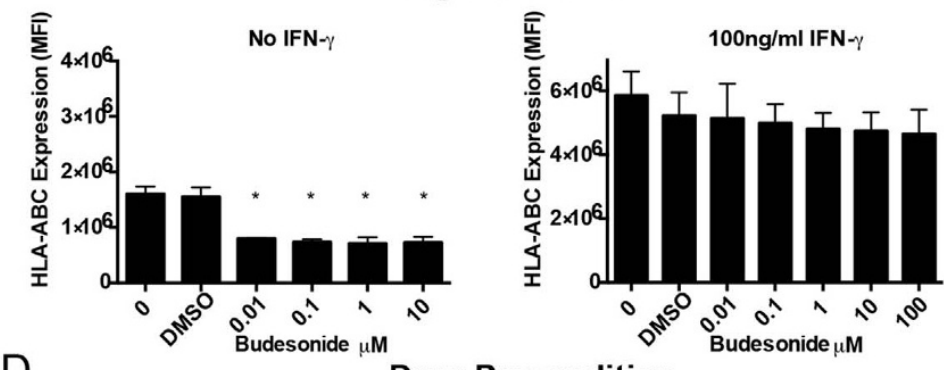

$\mathrm{D}$ Drug Precondition
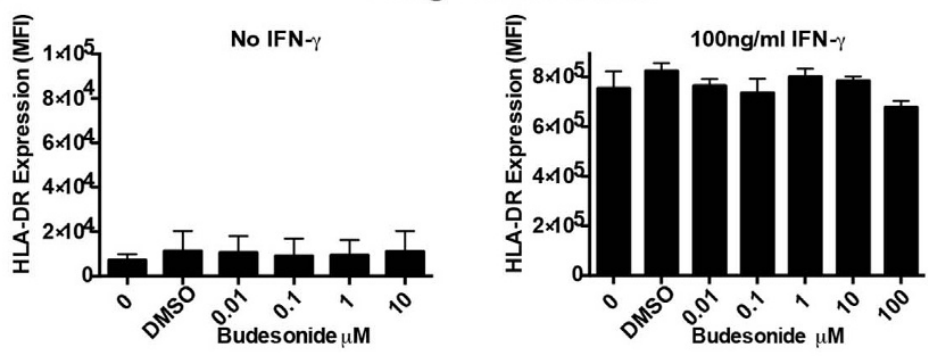

E
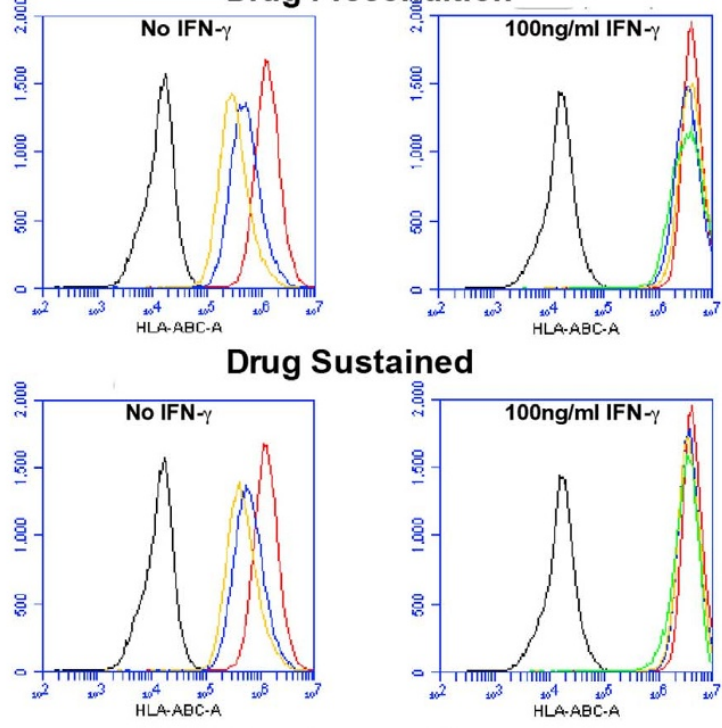

Drug Sustained

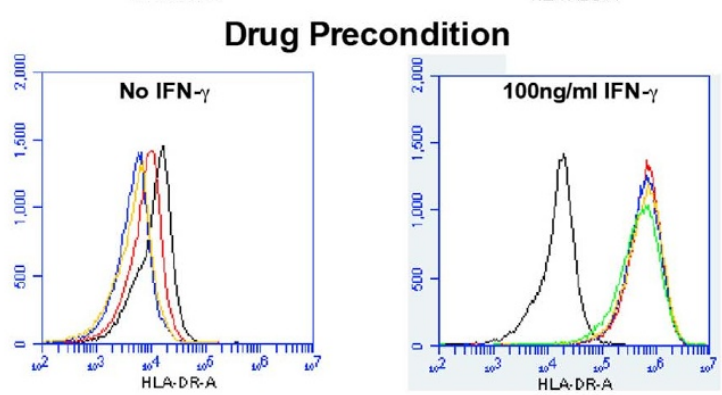

Drug Sustained
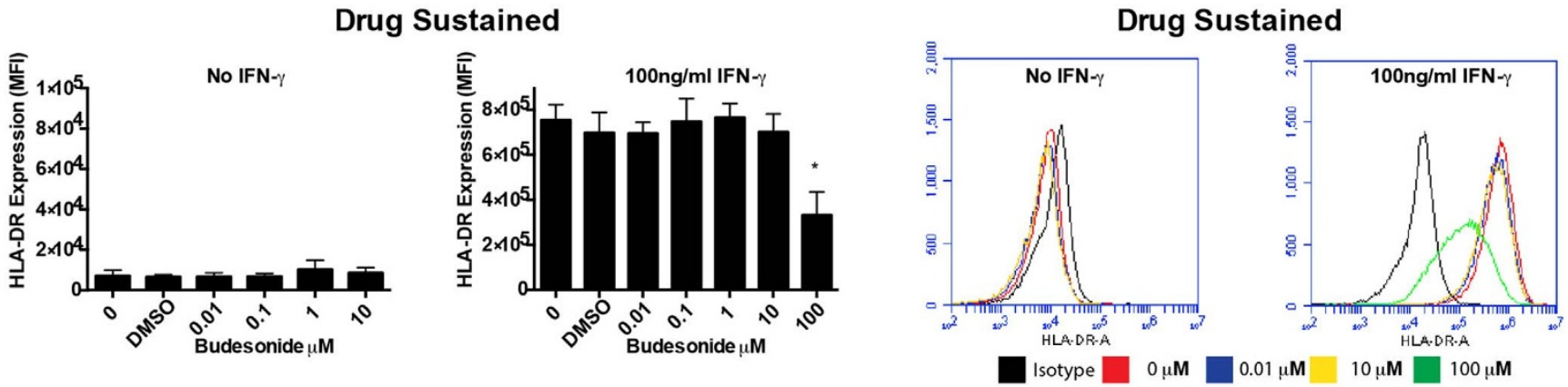

Figure $2 \mid$ Impact of budesonide on relative MHC molecule expression (A) Timing of MSC exposure to budesonide and IFN- $\gamma$ in the Drug Precondition and Drug Sustained groups. Flow cytometric analysis of (B,C) HLA-ABC and (D,E) HLA-DR surface expression of MSCs. MSC were plated in $25 \mathrm{~cm}^{2}$ flasks (100,000 MSC/flask) with media containing vehicle or budesonide and were either stimulated with IFN- $\gamma$ or left unstimulated. MSC were then cultured an additional 48 hours without budesonide (Drug Precondition) or with budesonide (Drug Sustained). (B,D) show mean fluorescence intensity (MFI) for each condition. (Bars are mean \pm SEM, One-way ANOVA with Bonferroni correction for multiple comparisons, $\mathrm{n}=3,{ }^{*} \mathrm{p}<0.001$ compared to untreated control). (C,E) show representative flow cytometry plots including isotype controls.

treatment) of the three donors, was used for all subsequent experiments. As the immunomodulatory function of IDO is dependent on its activity as an enzyme, an enzymatic activity assay was performed. Specifically, MSCs were grown according to the protocol depicted in Fig. 3A, collected, lysed to isolate IDO from the cytoplasm, and the quantity of L-kynurenine produced was measured by a colorimetric assay. Exposure to budesonide had a dramatic effect on MSC's IDO activity. While IDO expression naturally increases upon stimulation with IFN- $\gamma$, additional conditioning by budesonide resulted in an over 4 -fold increase in IDO activity (Fig. 3G).
Mechanism of IDO augmentation. To begin to elucidate the mechanism of budesonide-mediated enhancement of MSC IDO expression, we examined the timing and activation of intracellular pathways likely to be involved. Elevated IDO levels were first detected by western blot 24 hours after IFN- $\gamma$ exposure (Fig. 4A,B), consistent with previous reports that show IFN- $\gamma$ stimulates new transcription of IDO mRNA in MSCs $^{6}$. Furthermore, the effect does not appear to be mediated through increased sensitivity to IFN- $\gamma$, as the timing and degree of Stat- 1 phosphorylation between untreated (Fig. 4A) and budesonide treated (Fig. 4B) MSCs were 


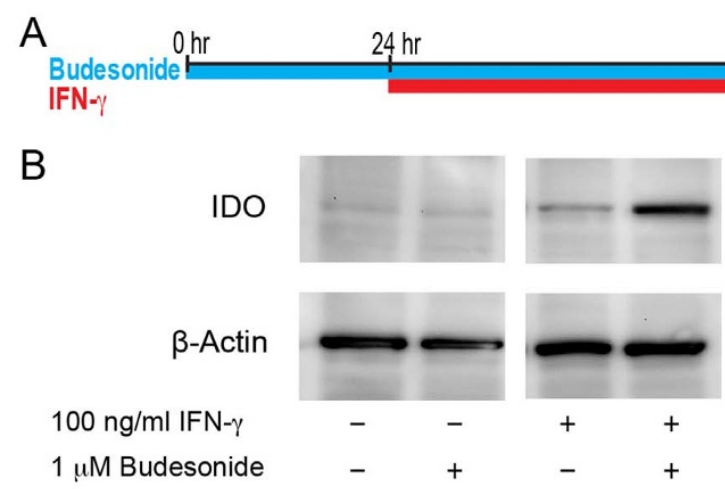

C
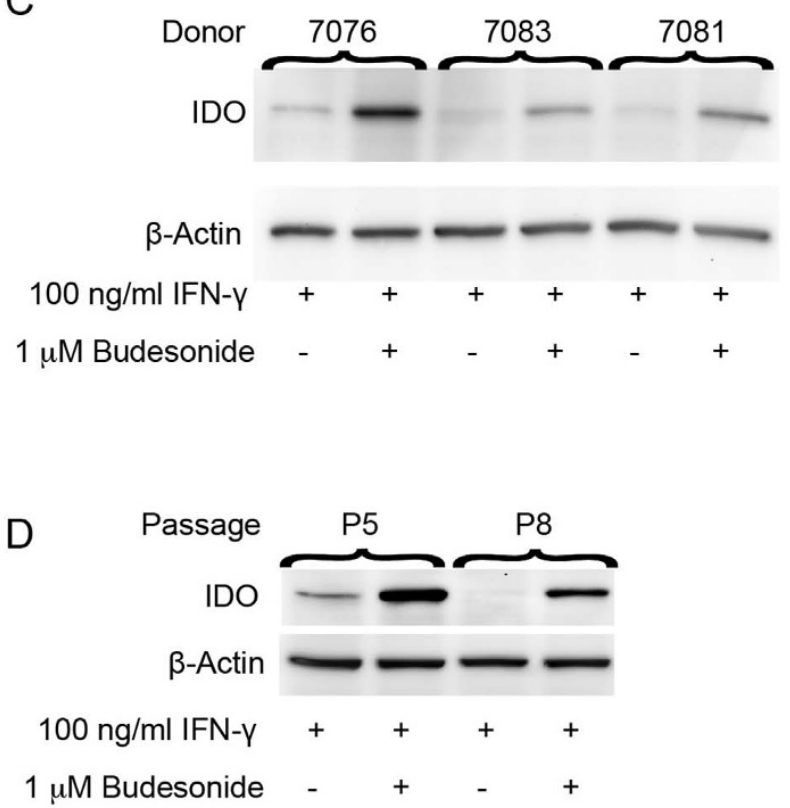

$\mathrm{E}$
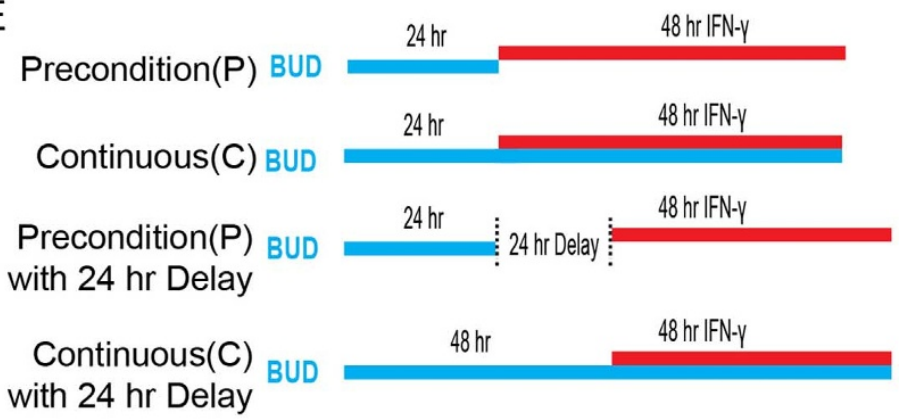

$\mathrm{F}$
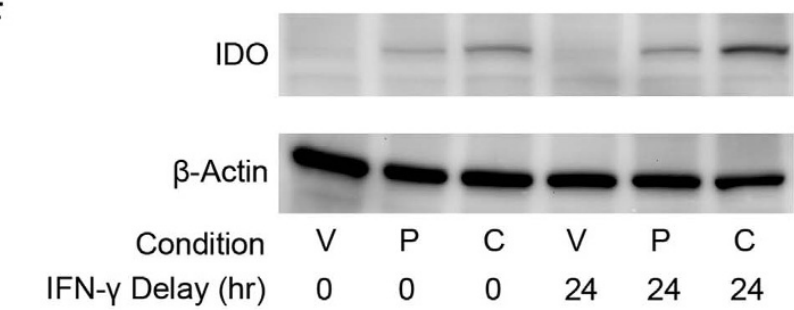

G

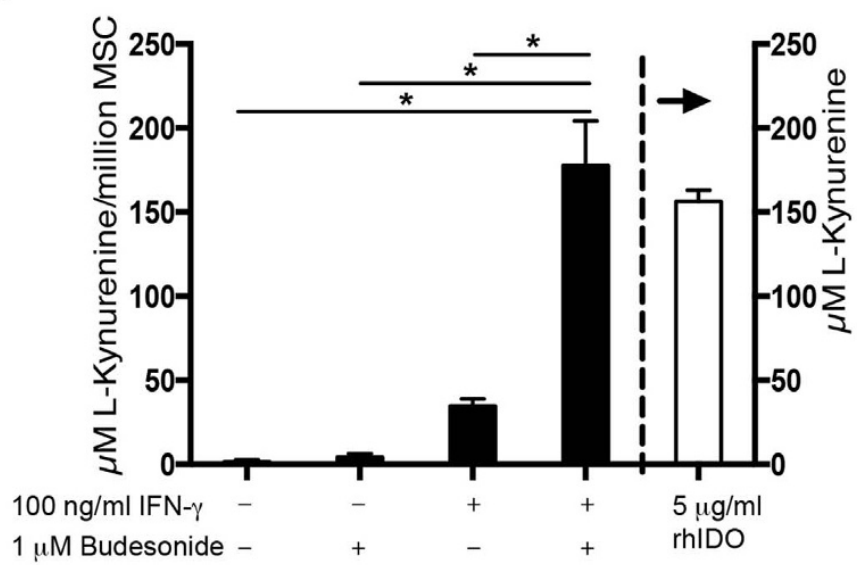

Figure 3 IDO expression is maximized following IFN- $\gamma$ and budesonide exposure. (A) Timing of MSC exposure to budesonide and IFN- $\gamma$. (B) Western blot of IDO protein content measured from MSC exposed to $1 \mu \mathrm{M}$ budesonide and $100 \mathrm{ng} / \mathrm{ml} \mathrm{IFN}-\gamma$. $\beta$-actin shown as loading control. (C) Western blot of IDO protein content measured from MSCs harvested from three independent donors exposed to $1 \mu \mathrm{M}$ budesonide and $100 \mathrm{ng} / \mathrm{ml}$ IFN- $\gamma$. $\beta$-actin shown as loading control. (D) P5 and P8 MSCs were treated with vehicle or budesonide for 24 hours and then additionally with $100 \mathrm{ng} / \mathrm{ml}$ IFN- $\gamma$ for 48 hours. Cell lysate was collected and analyzed for IDO content by western blot. (E) Impact of IFN- $\gamma$ and budesonide on the enzymatic activity of IDO harvested from MSC (donor 7083) lysate measured by production of kynurenine. $5 \mu \mathrm{g} / \mathrm{ml}$ rhIDO was used as an internal control for the assay(right axis). (Bars are mean \pm SEM One-way ANOVA with Tukey correction for multiple comparisons, $\mathrm{n}=3$, ${ }^{*} \mathrm{p}<0.001$ ). (F) Timing of MSC exposure to budesonide and IFN- $\gamma$. IFN- $\gamma$ was applied immediately following budesonide treatment or following a 24 hour delay in the preconditioning groups. (G) Western blot of IDO protein content measured from MSCs exposed to [V] DMSO vehicle, or [P] preconditioned, or [C] continuously exposed to $1 \mu \mathrm{M}$ budesonide. A 0 or 24 hour delay was introduced between the 24 hour budesonide preconditioning and exposure to IFN- $\gamma$. $\beta$-actin shown as loading control.

nearly identical. In addition, the pattern of immediate increase in phosphorylated Stat- 1 following IFN- $\gamma$ exposure and an increase in total Stat-1 following 24 hours of IFN- $\gamma$ treatment is consistent with patterns described in the literature for human cell lines ${ }^{32-34}$. Next we examined if budesonide was exerting its effect on IDO expression through the glucocorticoid receptor. Blocking the glucocorticoid receptor with $2 \mu \mathrm{M}$ of RU486 reversed budesonide's enhancing effect on IDO expression back to baseline levels (Fig. 4C). This data suggests budesonide mediated enhancement of IDO is dependent on the glucocorticoid receptor. Consistent with this observation is the finding that treatment with either budesonide or dexamethasone, both glucocorticoid steroids, results in similar enhancement in IDO expression (Fig. 4D). Interestingly, the promoter region for the IDO gene does not contain any known glucocorticoid responsive elements (GRE). Thus, steroid enhancement of
IDO is likely mediated through up-regulation of a glucocorticoid responsive intermediary that enhances the transcription of IDO. Therefore we looked for transcription factors known to bind the IDO promoter that are also sensitive to glucocorticoid steroids. IDO has previously been reported to be promoted by $\mathrm{FOXO}^{17}$, a transcription factor which has recently been shown to be a target of the glucocorticoid receptor ${ }^{35}$. To determine FOXO3's role in budesonide-mediated enhancement of MSC IDO, we performed siRNA knockdown experiments. MSCs were transfected with FOXO3 siRNA or scramble siRNA as a control and then treated with budesonide for 24 hours, after which cells were treated with IFN- $\gamma$. FOXO3 expression was measured 6 hours after IFN- $\gamma$ addition (preliminary experiments revealed peak FOXO3 content at this time point) and IDO was measured 30 hours after IFN- $\gamma$ addition. Transfection of MSCs with FOXO3 siRNA resulted in 
Vehicle Treated MSCs

Hours after IFN- $\gamma$ exposure

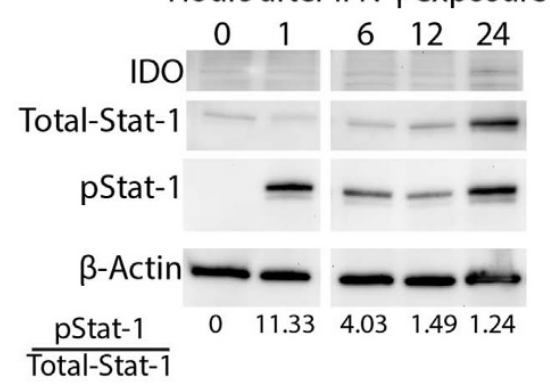

B

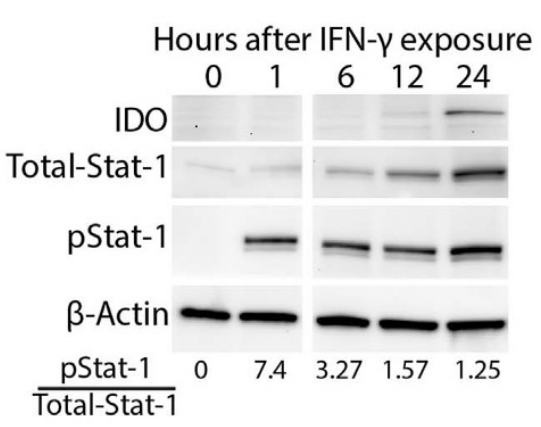

C

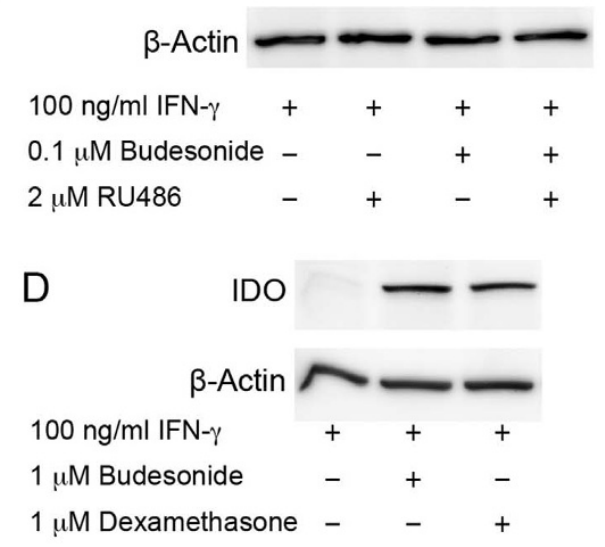

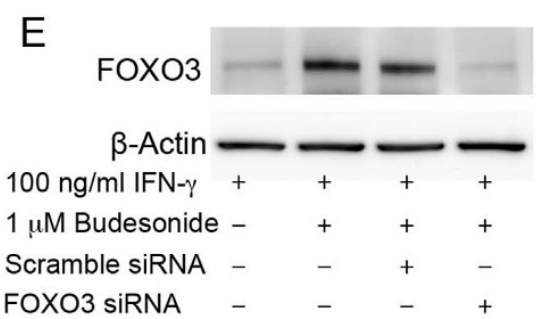

$\mathrm{F}$

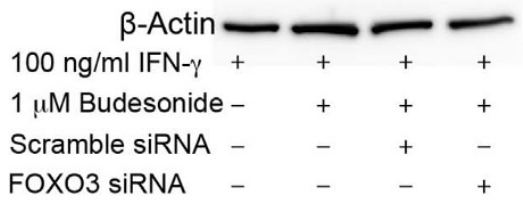

G

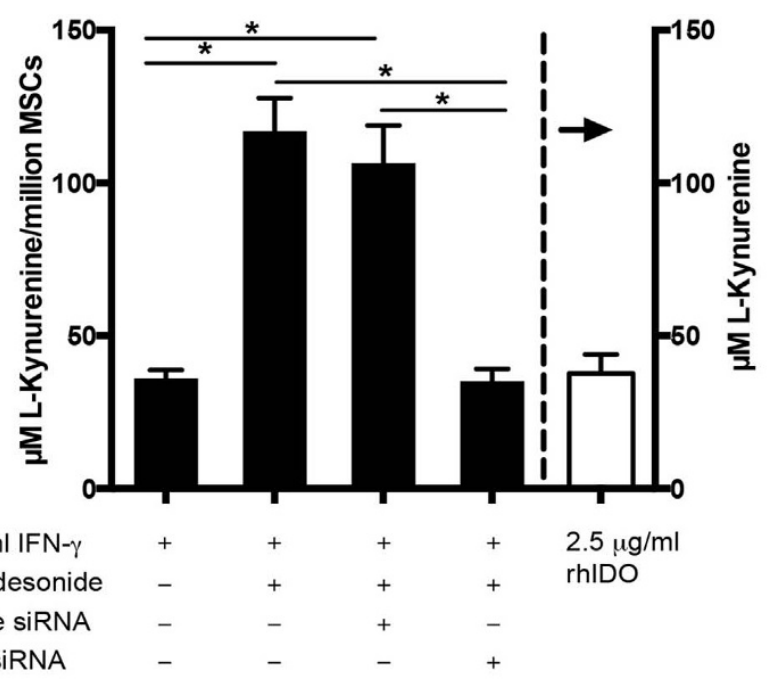

Figure $4 \mid$ Budesonide enhancement of IDO occurs through glucocorticoid receptor mediated upregulation of FOXO3 and not increased sensitivity to IFN- $\gamma$. MSCs were treated with (A) vehicle only or (B) $1 \mu \mathrm{M}$ budesonide for 24 hours and then additionally exposed to $100 \mathrm{ng} / \mathrm{ml}$ IFN- $\gamma$ (continuous exposure to vehicle or budesonide). Lysates of (A) untreated (vehicle only) MSCs and (B) $1 \mu \mathrm{M}$ budesonide treated MSCs were collected at 0, 1, 6, 12, and 24 hours after IFN- $\gamma$ exposure. To determine the timing of IDO up-regulation IDO protein content was measured at each time point. To determine if budesonide enhanced MSC sensitivity to IFN- $\gamma$, the degree of Stat-1 phosphorylation was measured as stat-1 phosphorylation is classically induced by IFN- $\gamma$ stimulation. $\beta$-actin shown as loading control. (bottom) Ratio of pStat- 1 to total Stat-1 is shown for each time point. (C) Inhibition of the glucocorticoid receptor with RU486 reverts IDO levels back to baseline levels in budesonide treated MSCs. (D) Western blot of IDO protein content measured from IFN- $\gamma$ stimulated MSC exposed to DMSO vehicle, budesonide, or dexamethasone. $\beta$-actin shown as loading control. To test the dependence of enhanced IDO expression on FOXO3, siRNA knockdown was performed. MSCs were transfected with FOXO3 siRNA or scrambled siRNA for 5 hours and then treated with budesonide for 24 hours followed by IFN- $\gamma$ treatment. Cell lysates were collected 6 and 30 hours following addition of IFN- $\gamma$ and analyzed for (E) FOXO3 and (F) IDO respectively. To confirm that inhibition of FOXO3 prevents enhanced expression of IDO, an IDO activity assay was performed. (G) Activity of IDO harvested from lysates of vehicle, budesonide, budesonide/FOXO3 siRNA, and budesonide/scrambled siRNA treated MSCs 48 hours after IFN- $\gamma$ stimulation. (Bars are mean \pm SEM, One-way ANOVA with Tukey correction for multiple comparisons, $\mathrm{n}=3$, $\left.*_{\mathrm{p}}<0.01\right)$.

nearly complete inhibition of FOXO3 (Fig. 4E) and reverted budesonide treated MSC IDO expression (Fig. 4F) and activity (Fig. 4G) back to the level of untreated MSCs. Collectively this data suggests budesonide's effect on MSC IDO expression is mediated by glucocorticoid-induced expression of FOXO3 which then acts as a genomic enhancer to augment IDO expression.

Establishing prolonged control of MSCs. We next sought to examine if we could prolong enhanced MSC IDO activity without the need for continuous exposure to soluble budesonide. Glucocorticoid steroids are commonly used clinically and potentially could be coadministered with MSCs, however, their lack of specificity causes them to have numerous off-target effects that can be detrimental to the overall health of a patient ${ }^{27,36,37}$. Thus, developing a method to control MSC phenotype without requiring co-administration of systemic glucocorticoid steroids thereby minimizing systemic exposure is desirable. To achieve prolonged control of MSC IDO activity we drew from our prior experience loading MSCs with drug loaded poly(lactic-co-glycolic acid) (PLGA) microparticles ${ }^{38}$. Previously we have shown that using the particle-in-cell approach, $1 \mu \mathrm{m}$ particles can be efficiently internalized into MSCs and remain stable for at least 7-days during which time, small molecule drugs can be released to influence the phenotype of cells ${ }^{38-40}$.

Budesonide microparticles $\sim 1 \mu \mathrm{m}$ in diameter with low polydispersity were formulated to control MSC IDO activity (Fig. 5A,B). Surface modification of the particles with poly-L-Lysine resulted in a 
zeta potential of $+10 \mathrm{mV}$ (Fig. $5 \mathrm{~B}$ ), which we have previously shown enhances particle uptake ${ }^{39}$. Budesonide was extracted from the particles and the drug loading (7.05\%, Fig. 5B) and encapsulation efficiency (51.2\%, Fig. 5B) were determined by HPLC. $10 \mathrm{kDa}$ molecular weight PLGA was used to formulate the particles to ensure rapid release of the drug during the first week following MSC particle modification. Release kinetic experiments were performed, revealing a burst release of $20 \%$ of total drug in the first 12 hours followed by a continuous release, with $\sim 80 \%$ of total drug released by day 10 (Fig. 5C). MSC internalization of particles was optimized as previously described ${ }^{39}$ by modulating the size and zeta-potential of the particles. MSC association with $1 \mu \mathrm{m}$ PLGA particles coated with poly-L-Lysine was confirmed by flow cytometry showing nearly all MSCs associated with particles (Fig. 5D). Confocal imaging revealed PLGA particles were not merely associating with the outer plasma membrane, but internalized (Fig. 5E). MSC's were engineered with BUD-Particles without impacting cell viability (Fig. 5F).

To test if the intracellular release of budesonide from PLGA particles would work similarly to soluble budesonide, we examined the expression and activity of IDO within cell lysates. MSCs were modified with either BUD-PLGA or Blank-PLGA particles overnight, allowed to rest 24 hours, and then stimulated with IFN- $\gamma$ for 48 hours, after which cells were harvested for analysis (Fig. 6A). As with soluble budesonide, BUD-PLGA particle modification significantly increased the content of IDO in MSC lysate (Fig. 6B). In addition, when compared head to head, BUD-PLGA particle modified MSCs expressed higher levels of IDO than MSCs simply pretreated with soluble budesonide before IFN- $\gamma$ activation (Fig. 6C). Finally, BUD-PLGA modified MSCs exhibited a $5 \times$ augmentation in IDO enzymatic activity over Blank-PLGA modified and unmodified
MSCs (Fig. 6D), an effect similar to what was observed with soluble budesonide (Fig. 3G).

Enhanced immunosuppressive properties in vitro. To test our hypothesis that enhanced IDO activity would lead to enhanced immunosuppression, MSCs were co-cultured with CD3/CD28 Dynabead activated PBMCs. BUD-Particle modified MSCs showed significantly enhanced suppression over budesonide preconditioned MSCs, which showed no advantage over naïve MSCs (Fig. 6E). To determine the degree of BUD-Particle modified MSC enhancement over naïve MSCs, ratiometric MSC:PBMC co-cultures were established by fixing the number of PBMCs in each well and varying the number of MSCs plated to achieve MSC:PBMC ratios of $1: 4,1: 8$, or $1: 16$. Unmodified, Blank-Particle modified, and BUD-Particle modified MSCs each suppressed PBMC proliferation in a cell-dose dependent manner. However, BUD-Particle MSCs showed enhanced suppression of PBMCs at all ratios tested as evidenced by reduced proliferation (Fig. 7C,D) and decreased IFN$\gamma$ production (Fig. 7E). Impressively, BUD-Particle MSC co-cultures at $1: 8$ and $1: 16$ ratios were as effective at suppressing PBMC proliferation and IFN- $\gamma$ secretion as native MSCs at $1: 4$ and $1: 8$ ratios, respectively. In other words, twice as many unmodified MSCs are required to achieve an equivalent in vitro suppressive effect as BUD-Particle MSCs.

As IDO activity is dependent on IFN- $\gamma$ stimulation, we hypothesized the effect could be further accentuated by pre-activation of MSCs. Activating MSCs with IFN- $\gamma$ prior to the co-culture enhances IDO expression of the MSCs to maximize potency at the beginning of the assay. This is akin to activating MSCs prior to administration to a patient to maximize potency without relying on the recipient for
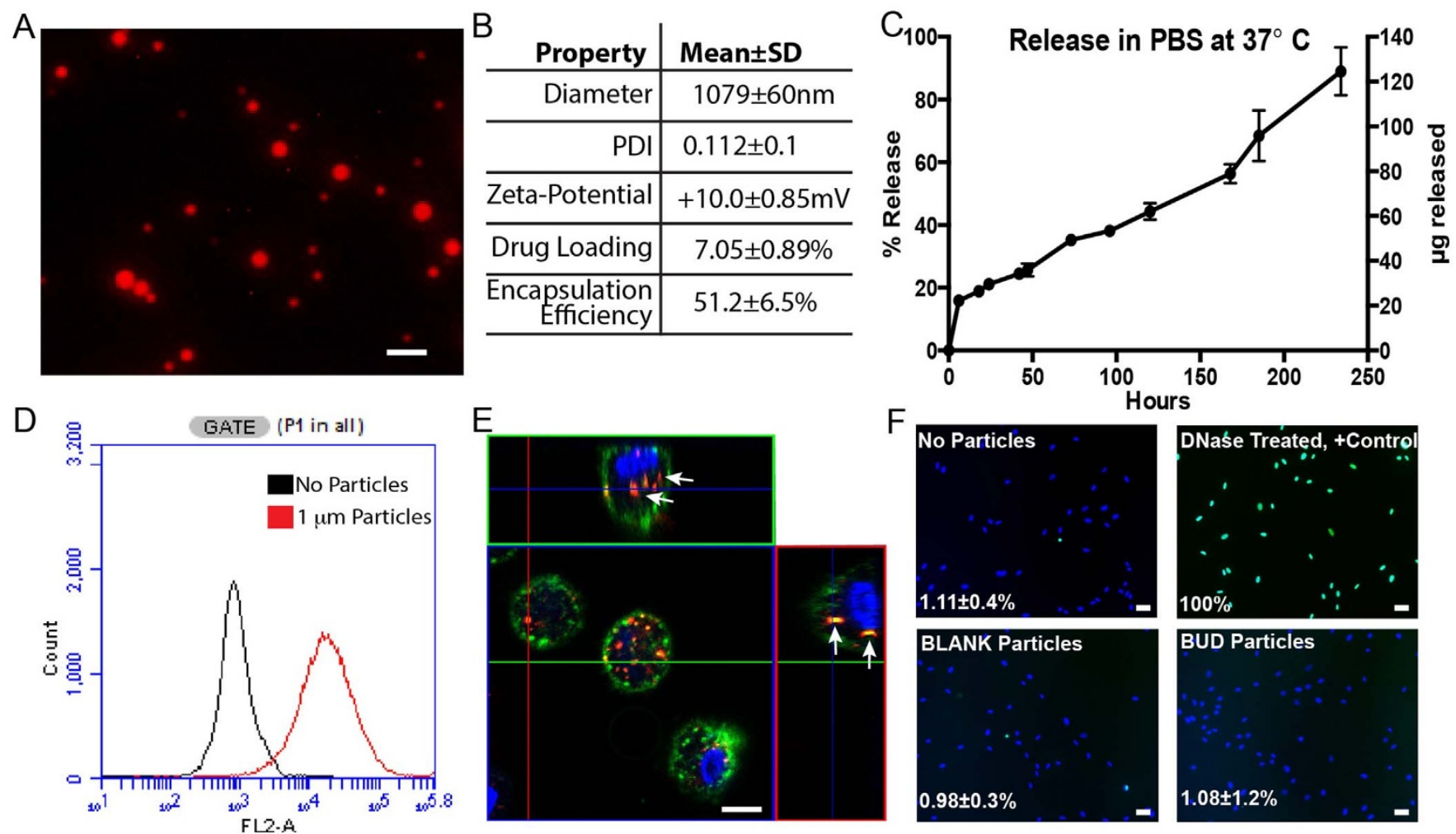

Figure 5 | Budesonide particle modification to enable sustained control of MSC phenotype. (A) Fluorescent image of DiI labeled Budesonide PLGA microparticles(Scale bar $5 \mu \mathrm{m}$ ). (B) Physicochemical properties of budesonide PLGA microparticles. (C) Release kinetics of budesonide from 2 mg of PLGA microparticles into PBS at $37^{\circ} \mathrm{C}$. (D) MSC association with $1 \mu \mathrm{m}$, PLL coated particles was assessed by flow cytometry (Representative plot). (E) Representative confocal image of an MSC modified with $1 \mu \mathrm{m}$ diameter PLGA particles (white arrows point to red particles) revealing particles are predominately intracellular rather than membrane associated (membrane stained Green, nuclei shown in Blue, scale bar $10 \mu \mathrm{m}$ ). (F) Particle modified MSC viability examined by TUNEL staining. TUNEL stain shown in green with nuclei counterstained with Hoechst (Blue). (bottom) Percent apoptotic cells (mean $\pm \mathrm{SD}$, TUNEL $+/$ Hoechst + nuclei, $\mathrm{n}=3$ ) shown in each image (Scale bar $50 \mu \mathrm{m})$. 


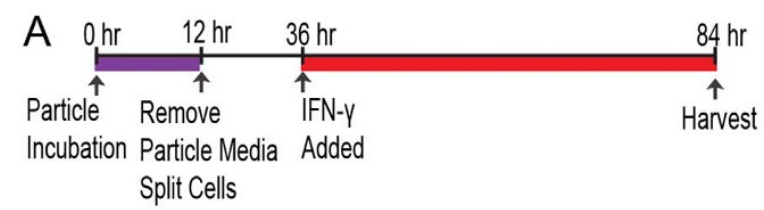

B

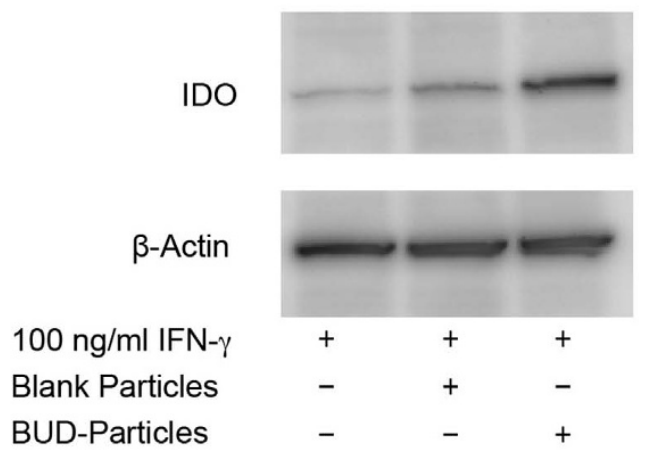

C

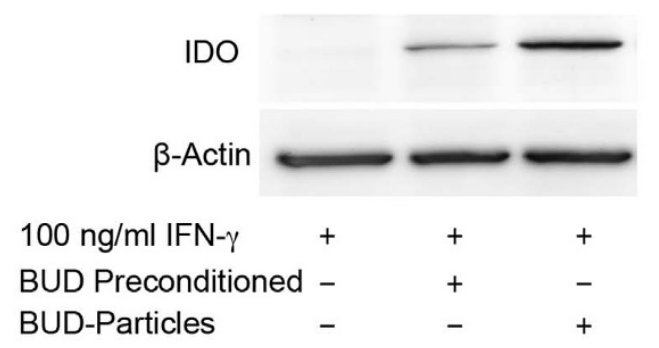

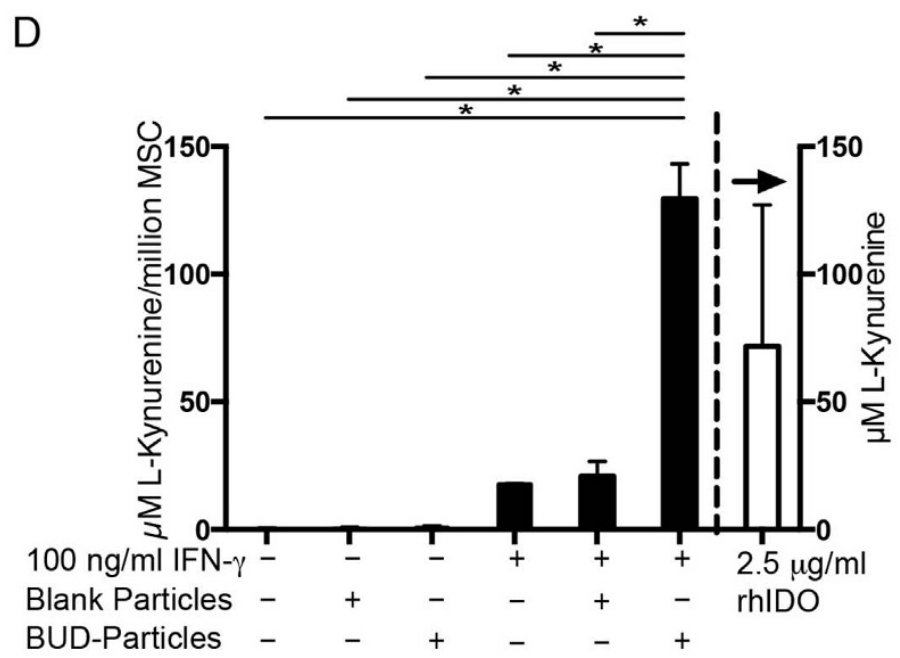

$\mathrm{E}$

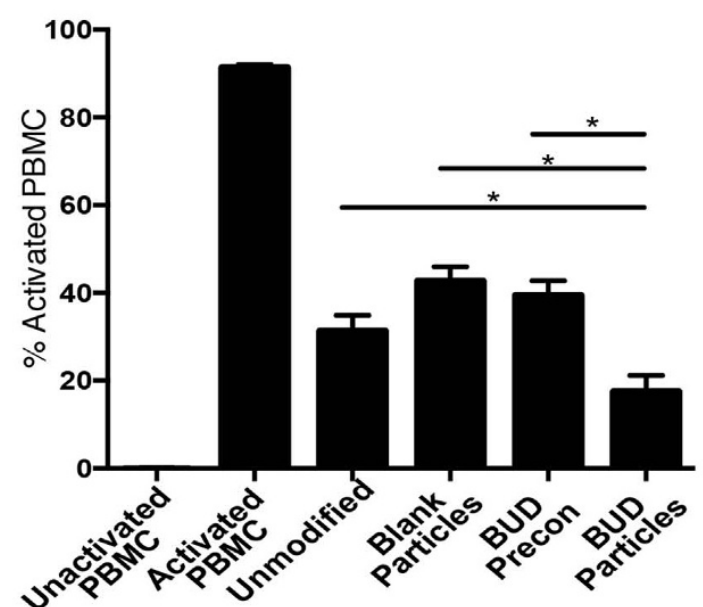

Figure 6 Budesonide particles enhance MSC immunomodulatory potential. (A) Timing of MSC particle modification and exposure to IFN- $\gamma$. MSCs were modified with particles overnight, harvested and split into new flasks and then exposed to IFN- $\gamma$ for 48 hours. (B) Western blot of IDO protein content measured from unmodified, Blank-Particle modified, or BUD-Particle modified MSCs exposed to $100 \mathrm{ng} / \mathrm{ml}$ IFN- $\gamma$. $\beta$-actin shown as loading control. (C) IDO content following 48 hours of IFN- $\gamma$ stimulation in untreated, budesonide preconditioned, and budesonide-particle modified MSCs. $\beta$-actin shown as loading control. (D) Impact of BUD-Particle modification on MSC IDO activity with and without stimulation with 100 ng/ml IFN- $\gamma$. IDO activity measured by the production of L-kynurenine from generated from MSC lysate incubated with tryptophan. $5 \mu \mathrm{g} / \mathrm{ml} \mathrm{rhIDO} \mathrm{was} \mathrm{used}$ as an internal control for the assay(right axis). (Bars are mean \pm SEM, One-way ANOVA with Tukey correction for multiple comparisons, $\mathrm{n}=3$, ${ }^{*} \mathrm{p}<0.001$ ). (E) Bud-particle engineered MSCs exhibit enhanced suppression over budesonide preconditioned MSCs. Quantification of MSC suppression of PBMCs after 5 day MSC:PBMC co-cultures. Data represents average of three experiments conducted with independent PBMC donors. Un-stimulated control for each donor used to set threshold for PBMC activation. (Bars are mean \pm SEM, Ordinary One-way ANOVA with Tukey correction for multiple comparisons, $\left.\mathrm{n}=3,{ }^{*} \mathrm{p}<0.05\right)$.

activation. Thus, the MSC:PBMC co-cultures were repeated with MSCs preconditioned with IFN- $\gamma$ for 48 hours to stimulate IDO expression prior to plating in the co-cultures. Pre-activation of MSC IDO expression resulted in similar trends in unmodified MSCs, inferior suppression by Blank-Particle MSCs, and further enhanced BUD-Particle MSCs ability to suppress PBMC proliferation (Fig. 7F,G) and IFN- $\gamma$ secretion (Fig. 7H) compared to co-cultures with unactivated MSCs.

Next we sought to examine the mechanism responsible for the enhanced suppression. Soluble budesonide released from the MSCs did not appear to be responsible, as PBMCs treated with $1 \mu \mathrm{M}$ and $10 \mu \mathrm{M}$ budesonide (no MSCs) were activated to the same degree as untreated PBMCs (Fig. 8A). In order to determine if the enhanced suppressive effect of BUD-Particle modified MSCs can be attributed to IDO or that of other soluble factors, we used a widely used inhibitor of IDO, 1-methyl-DL-tryptophan (1-MT) to inhibit IDO activity. MSC:PBMC co-cultures were repeated at a MSC:PBMC ratio of $1: 8$ with or without the addition of $1 \mathrm{mM} 1-\mathrm{MT}$. Inhibition of IDO with 1-MT completely abolished BUD-Particle MSCs inhibitory effect suggesting IDO is responsible for the enhanced immunomodulatory potency of BUD-Particle MSCs (Fig. 8B).

\section{Discussion}

Herein we have demonstrated MSC immunomodulatory performance can be enhanced through intracellular delivery of steroids. Budesonide treatment resulted in an over 4-fold increase in IFN- $\gamma$ stimulated MSC IDO activity. Furthermore, budesonide treatment enhanced the expression of IDO in MSCs from multiple donors and restored IDO expression in over-passaged MSCs providing a tool to address donor-donor and passage-passage variability in MSC potency. The enhancement of MSC IDO expression was not limited to budesonide, as similar levels of enhancement were also observed with dexamethasone treatment. Unlike other cell types such as fibroblasts $^{29}$ and lymphocytes ${ }^{41}$, MSC viability, metabolic activity, and morphology, were not significantly impacted by exposure to a wide range of budesonide concentrations. In addition to enhancement of 
A
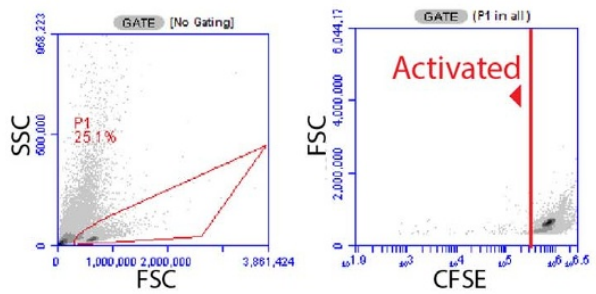

C

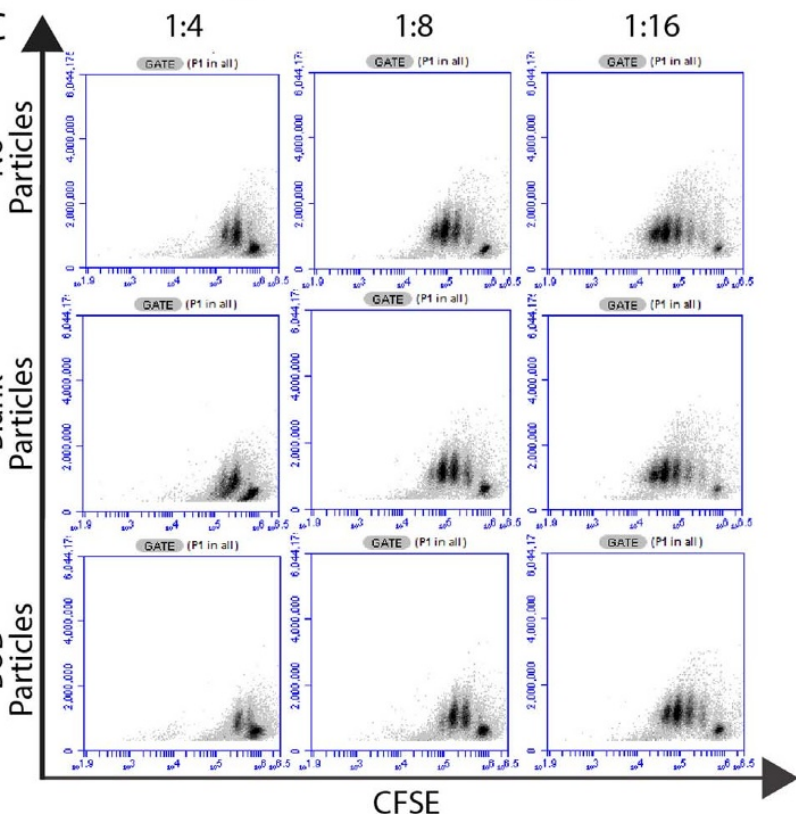

$\mathrm{F}$

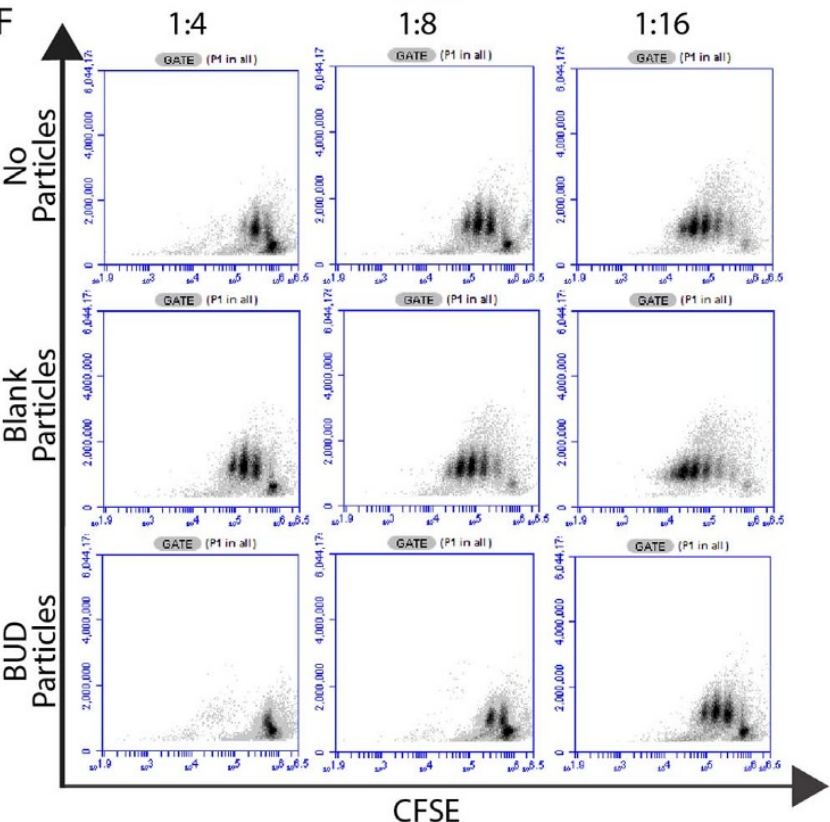

B
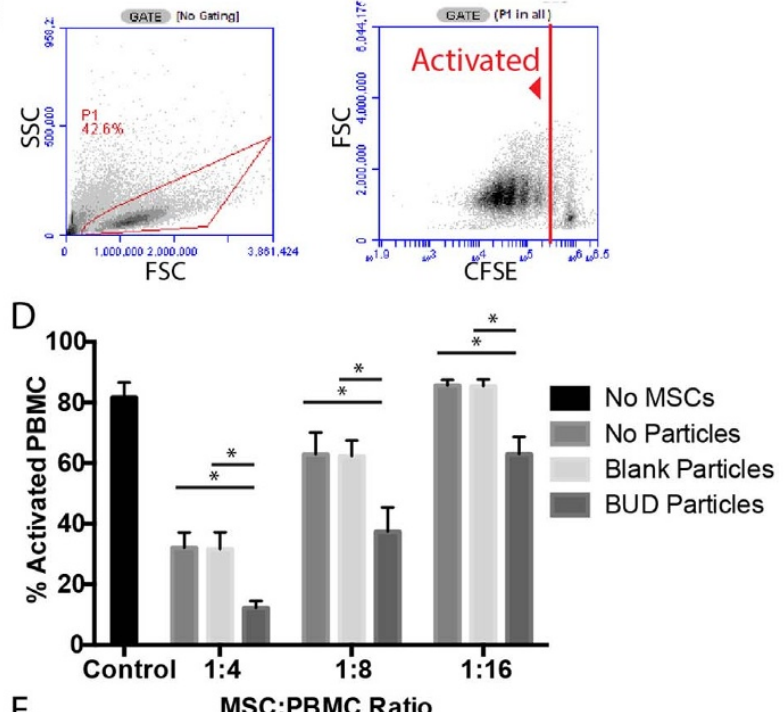

E
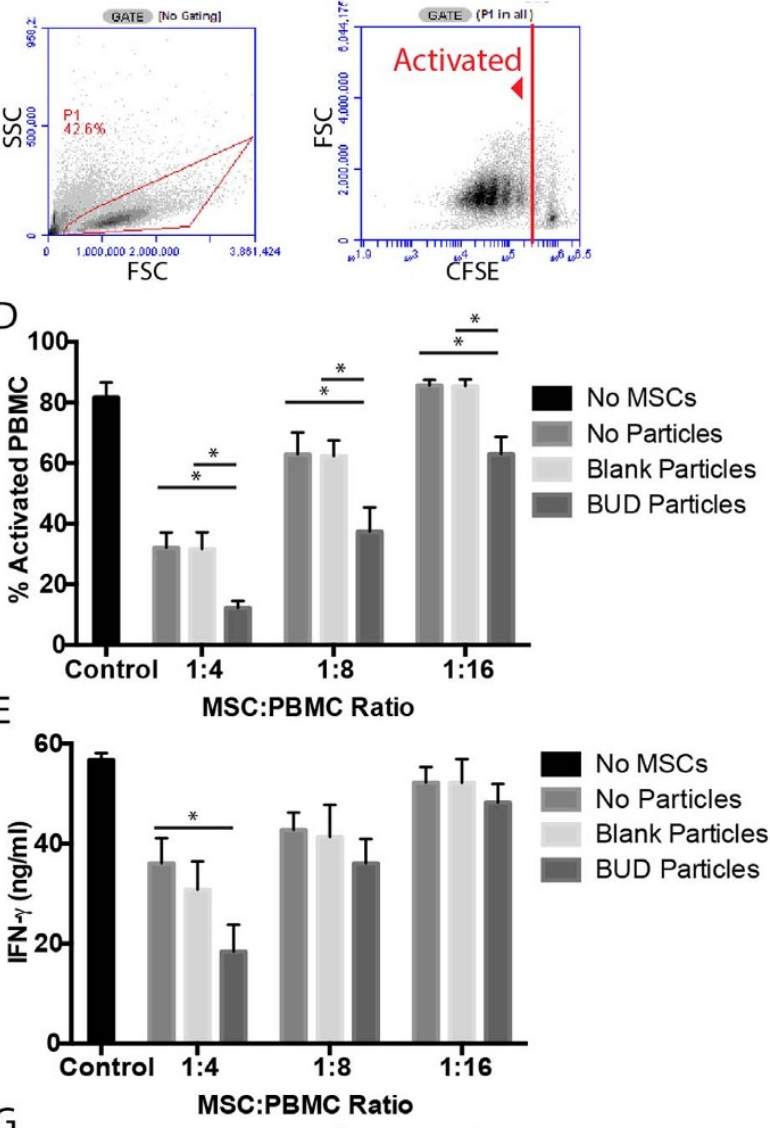

$G$

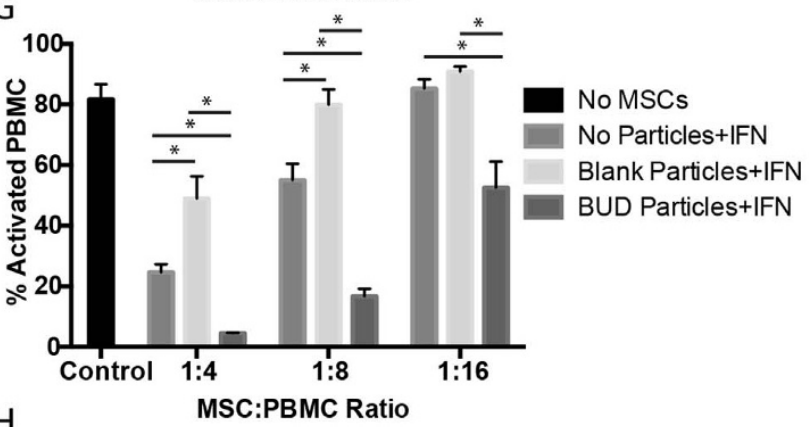

H

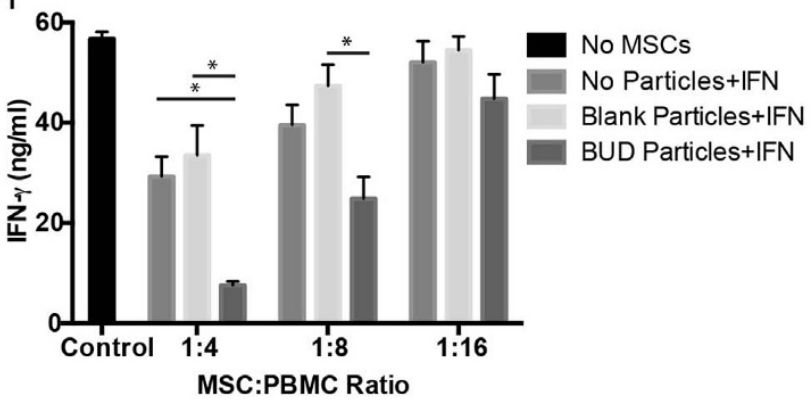

Figure $7 \mid$ Engineered MSCs exhibit enhanced immunomodulatory potency. Gating and representative flow cytometry plot of (A) unstimulated and (B) CD3+/CD28 + Dynabead stimulated CFSE stained PBMCs (CFSE vs. Forward Scatter). (C) Representative CFSE vs forward scatter flow cytometry plots of PBMCs harvested from 5 day MSC:PBMC co-cultures. MSC:PBMC ratio and type of MSC particle modification are listed on each column and row respectively. As PBMCs are activated and divide, CFSE is diluted $1: 2$, resulting in discrete daughter generations that shift to the left with each cell division. (D) Quantification of MSC suppression of PBMCs harvested from three independent donors. Un-stimulated control for each donor used to set threshold for PBMC activation. (E) IFN- $\gamma$ concentration measured from supernatant of MSC:PBMC co-cultures as marker of PBMC activation. (F) Representative CFSE vs. forward scatter flow cytometry plots of PBMCs harvested from 5 day MSC:PBMC co-cultures containing MSCs preconditioned with IFN- $\gamma$ to stimulate IDO activity. MSC:PBMC ratio and type of MSC particle modification are listed on each column and row respectively. (G) Quantification of MSC suppression of PBMCs harvested from three independent donors. Un-stimulated control for each donor used to set threshold for PBMC activation. (H) IFN- $\gamma$ concentration measured from supernatant of preconditioned MSC:PBMC co-cultures as a marker of PBMC activation. Un-stimulated PBMC controls showed no detectable secretion of IFN- $\gamma$. (Bars are mean \pm SEM, Two-way ANOVA with Tukey correction for multiple comparisons, $\left.\mathrm{n}=3,{ }^{*} \mathrm{p}<0.05\right)$. 
MSC IDO activity, we also observed a reduction in MSC expression of HLA-ABC at all doses in unstimulated conditions and a reduction in HLA-DR expression at high doses in IFN- $\gamma$ stimulated conditions. The reduced expression of MHC molecules due to budesonide treatment further minimizes the hypo-immunogenic phenotype of native MSC. However, following IFN- $\gamma$ stimulation the level of HLA-ABC and HLA-DR expression in budesonide treated MSC remains high, and is thus unlikely to significantly alter MSC rejection in allogeneic transplant settings. In fact, previous attempts to shield MSCs from allo-rejection through reduction of HLA-ABC expression required near complete blockade of HLA-ABC presentation in both unstimulated and IFN- $\gamma$ stimulated conditions by viral immunoevasins ${ }^{42,43}$. Nevertheless, approaches like this that reduce expression of $\mathrm{MHC}$ molecules should be explored further to reduce immunological events following allogeneic transplantation of MSC. Overall, budesonide treatment maintained MSCs proliferative capabilities while reducing MSC's immunophenotype and greatly enhancing MSC's immunomodulatory potency through increased IDO activity. To our knowledge, this is the first report of small molecule enhancement of IDO activity in MSCs and the approach represents a significant opportunity to augment MSC-based therapies.

Enhanced expression of IDO was shown to be dependent on both the glucocorticoid receptor and FOXO3, as inhibition of either abrogated the effect. In addition to the current report of FOXO3 enhancement of MSC immunosuppression, enhanced FOXO3 expression has also recently been reported to be a marker of tolerogenic dendritic cells ${ }^{44,45}$. FOXO3 expressing dendritic cells produce reduced levels of IL- $6^{44}$ and elevated levels of IDO $^{45}$. Inhibition of FOXO3 in tumor associated dendritic cells results in decreased levels of IDO and enhanced anti-tumor immune responses ${ }^{45,46}$. While these studies have examined the FOXO3-IDO pathway in the context of pathology, we believe there is great potential to leverage the tolerogenic effects to treat graft versus host disease, autoimmune conditions such as Crohn's and multiple sclerosis, and prevent rejection of transplanted tissues.

As the enhanced immunosuppressive phenotype of MSCs was most prominent in cultures continuously exposed to budesonide (Fig 3E,F), we employed an engineering strategy to establish prolonged control of MSCs. MSCs were engineered with budesonide loaded PLGA particles that continuously release budesonide, resulting in a 5-fold enhancement in IFN- $\gamma$ stimulated MSC IDO activity. BUD-Particle MSCs were shown to exhibit enhanced immunosuppressive potency in PBMC co-culture assays in an IDO dependent manor. Inhibition of IDO with 1-MT led to abrogation of MSCs' suppressive potential, implicating IDO as a primary mechanism of budesonide mediated MSC enhancement. BUD-Particle engineered MSCs were twice as potent in suppressing PBMC proliferation and IFN- $\gamma$ secretion, as determined from ratiometric co-culture experiments. Furthermore, pre-activating MSCs with IFN- $\gamma$ prior to the co-culture further enhanced their potency. A pre-activation approach could be attractive to maximize the systemic immunomodulatory effects of MSCs, where cells may not come in contact with sufficient levels of IFN- $\gamma$ to exert their activated systemic effects. In contrast, for local administration of MSCs, pre-activation is likely not necessary as the MSCs will be activated in situ at the site of inflammation. Enhancing the potency of a single MSC enables fewer MSCs to be administered to achieve the same therapeutic effect and enables a single cell to exert a significant impact on its microenvironment. Using the particle-in-cell platform also enables local control of MSC phenotype without requiring soluble glucocorticoid steroids, which in vivo, can contribute to systemic side effects. In addition, this technique can be used to augment the potency of MSCs harvested from different donors and tissues, eliminating the need to select MSCs from only donors or tissues that have high native immunosuppressive potential. Furthermore, as enhanced IDO activity has been shown to lead to tolerance in the setting of pregnancy ${ }^{18}$, solid organ transplantation ${ }^{47}$, and tumor evasion ${ }^{19}$, BUD-PLGA MSCs may be able to extend their therapeutic window by promoting tolerance and evading immune clearance.

In the current study we have demonstrated the utility of BUDParticle MSCs in in vitro co-cultures. However, the potential benefits of an MSC therapeutic with enhanced IDO expression could be far reaching. This strategy may be used to augment therapeutic potency of MSC therapies by suppressing active inflammation and inducing tolerance in the setting of GvHD, Crohn's disease, and transplant biology. In addition, modified MSCs with enhanced IDO activity could also be applied to prevent bio-fouling of orthopedic implants and other medical devices due to IDO's antimicrobial properties ${ }^{16}$. Adaptation of this platform to other cell types to enhance IDO expression (or other immune modulators) for exogenous cell therapy should be possible. We believe steroid preconditioning and especially intracellular engineering strategies present an opportunity to control the therapeutic potency of MSCs and expand the pool of potent cells that can be used for therapies. This may be useful to reduce the number of cells needed for each patient, and ultimately could lead to improved patient outcomes.

\section{Methods}

Culture of MSCs. Primary human MSCs were obtained from Texas A\&M Health Science Center, College of Medicine, Institute for Regenerative Medicine at Scott \& White Hospital which has a grant from NCRR of the NIH, Grant \#P40RR017447
A

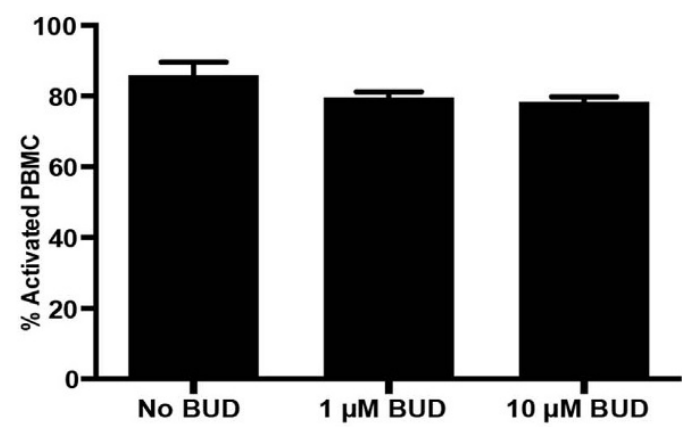

B

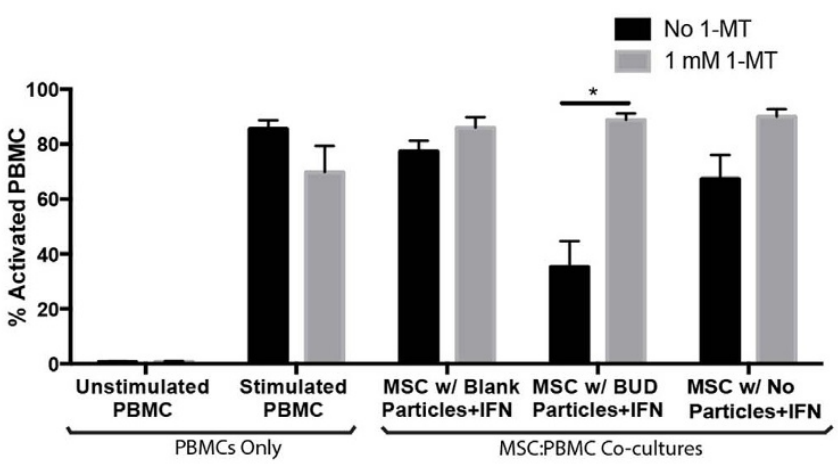

Figure 8 PBMC suppression is due to IDO and not soluble budesonide. (A) Budesonide alone does not suppress CD3/CD28 stimulated activation of PBMCs. Quantification of budesonide mediated suppression or PBMC harvested from three independent donors. Un-stimulated control for each donor used to set threshold for PBMC activation. (Bars are mean \pm SEM) (B) Inhibition of IDO abolishes MSC suppression of PBMCs. Quantification of MSC suppression of PBMCs harvested from three independent donors with and without addition of the IDO inhibitor 1-MT. Co-cultures performed with MSCs preconditioned with IFN- $\gamma$ to stimulate IDO activity at an MSC:PBMC ratio of 1:8. Un-stimulated control for each donor used to set threshold for PBMC activation. (Bars are mean \pm SEM, Two-way ANOVA with Tukey correction for multiple comparisons, $\mathrm{n}=3,{ }^{*} \mathrm{p}<0.05$ ). 
(Donors 7076, 7081, and 7083, informed consent gathered prior to cell collection) MSCs were maintained in cell-start (Invitrogen) coated flasks with StemPro MSC SFM culture media (Invitrogen) supplemented with 1\% (v/v) L-Glutamine (Invitrogen), and 1\% penicillin:streptomycin (Invitrogen). Cells were plated at a density of 6,000 cells $/ \mathrm{cm}^{2}$ and were passaged when flasks reached $70-80 \%$ confluence. All experiments were performed with passage 3-6 MSCs (Population doubling level between 4-8 from initial plating) unless indicated otherwise.

Metabolic activity, viability, and morphology assays. Metabolic activity of MSCs was assessed by XTT (ATCC) following 24, 48, or 72 hour exposure to budesonide. Briefly, 15,000 MSCs were plated in each well of a 96 well plate in $100 \mu \mathrm{l}$ of culture media containing DMSO vehicle and $0.001,0.01,0.1,1,10$, or $100 \mu \mathrm{M}$ budesonide. Cultures were maintained for 24,48 , or 72 hours in a humidified culture chamber. Culture media with no cells and wells with half the starting cell density were used as internal controls for each experiment. All conditions were explored in 5 wells for each experiment. To measure metabolic activity, $50 \mu \mathrm{l}$ of activated XTT reagent was prepared and added to each well per manufacturer's instructions. The plate was incubated for 3 hours and read at $450 \mathrm{~nm}$ and $630 \mathrm{~nm}$ for reference. To determine the morphology and viability of budesonide treated MSCs, 30,000 cells were plated into each well of a 24 well plate with vehicle or $0.001-100 \mu \mathrm{M}$ budesonide supplemented culture media. Cells were grown for 24,48 , or 72 hours. Before harvest, 4 random fields of each well were imaged at $10 \times$ using an inverted phase contrast microscope to capture cell morphology and differences in cell proliferation. To determine viability, cells were then harvested with Accutase, washed, and resuspended in $100 \mu \mathrm{l}$ stain solution containing $5 \mu \mathrm{l} / \mathrm{ml}$ FITC-Annexin-V and $1 \mu \mathrm{l}$ propidium iodide (Invitrogen) and stained on ice for $15 \mathrm{~min}$. Cells were washed with $400 \mu \mathrm{l}$ PBS, centrifuged, re-suspended in fresh PBS and analyzed by an Accuri C6 flow cytometer. MSCs treated with staurosporine to induce cell death were used as a positive control for each experiment. TUNEL staining of budesonide treated and particle modified MSCs was performed per manufacture's instructions using a in situ cell death detection kit-fluorescein (Roche) to label double strand DNA breaks indicative of apoptosis and counterstaining nuclei with Hoescht (Invitrogen). Fixed, DNase treated MSCs $(300 \mathrm{U} / \mathrm{ml}$ DNase, $1 \mathrm{mg} / \mathrm{ml} \mathrm{BSA}$, in $50 \mathrm{mM}$ TrisHCL for $10 \mathrm{~min}$ at room temperature) were used as a positive control for double strand DNA breaks. Image (NIH) was used to quantify the number of dual stained nuclei, all conditions were performed in triplicate.

Flow Cytometry. Expression of MHC molecules on MSCs following 24 hour preconditioning or continuous exposure to various doses of budesonide was determined using an Accuri C6 flow cytometer. For each condition, MSCs were grown in T25 flasks, harvested with Accutase, washed, and split for staining with either Alexa Fluor 488 anti-human HLA-ABC and Alexa Fluor 647 anti-human HLA-DR or isotype controls. Cells were re-suspended in antibody solution in PBS + $1 \%$ BSA and stained on ice for $15 \mathrm{~min}$. Cells were washed with $400 \mu \mathrm{l}$ PBS $+1 \%$ BSA, centrifuged, and analyzed by flow cytometry. Mean fluorescence intensity (MFI) was determined using CFlow software.

IDO Activity Assay. MSC cultures were grown to $80 \%$ confluence in T75 flasks. Cells were washed with PBS, harvested with Accutase cell dissociation reagent (Invitrogen), centrifuged, counted, and resuspended in $300 \mu \mathrm{l}$ of ice cold PBS(without $\mathrm{Ca}^{2+} / \mathrm{Mg}^{2+}$ ions) with $0.1 \%(\mathrm{w} / \mathrm{v})$ Triton X-100. Cell suspensions were lysed through triplicate freeze thaw cycles, and briefly pulse sonicated using a probe sonicator with power output of $3 \mathrm{~W}$. Lysates were centrifuged at $25000 \mathrm{rcf}$ at $4{ }^{\circ} \mathrm{C}$ for 30 minutes. $250 \mathrm{ul}$ of sample supernatant, recombinant human IDO (rhIDO), or L-kynurenine standard were mixed in a 1:1 ratio with $250 \mu \mathrm{l}$ of IDO buffer ( $40 \mathrm{mM}$ ascorbic acid, $20 \mu \mathrm{M}$ methylene blue, $200 \mu \mathrm{g} / \mathrm{ml}$ catalase, $800 \mu \mathrm{M}$ L-tryptophan, in $50 \mathrm{mM}$ MES buffer, pH 6.5). Samples were incubated at $37^{\circ} \mathrm{C}$ for $45 \mathrm{~min}$, followed by addition of $100 \mu \mathrm{l}$ of tricholoracetic acid $(30 \%, \mathrm{w} / \mathrm{v})$ and incubated at $52^{\circ} \mathrm{C}$ for $30 \mathrm{~min}$. Samples were centrifuged at $2500 \mathrm{rcf}$ for $10 \mathrm{~min}$ to remove proteins, and $100 \mu \mathrm{l}$ of the resultant supernatant for each sample was added to a 96 well plate (all samples and standards measured in duplicate). $100 \mu \mathrm{l}$ of Ehrlich's reagent ( $0.8 \%$ pdimethylaminobenzaldehyde in glacial acetic acid) was added to each well to induce a color change. Samples were incubated at room temperature for $10 \mathrm{~min}$ and then read on a microplate reader at $490 \mathrm{~nm}$. rhIDO was used as a positive control in each assay and an internal L-kynurenine standard $(8-5000 \mu \mathrm{M})$ was included to determine kynurenine production of IDO from MSC lysate.

siRNA Transfection. T25 flasks were seeded with 166,000 MSCs and incubated overnight at $37^{\circ} \mathrm{C}$. siRNA transfection was performed as follows. $10 \mathrm{ul}$ of Lipofectamine RNAiMAX transfection reagent (Invitrogen) per sample was diluted into $0.5 \mathrm{ml}$ of Opti-MEM media (Invitrogen), and gently mixed. $20 \mu \mathrm{l}$ of SignalSilence FOXO3 siRNA (Cell Signaling Technology, \#6302S) or scramble control siRNA (Cell Signaling Technology, \#6201S) per sample was diluted into $0.5 \mathrm{ml}$ of Opti-MEM media and gently mixed. Both solutions were incubated at room temperature for five minutes. The solutions were then gently mixed and incubated for twenty minutes at room temperature in the dark. During this time, the cells were washed with PBS-/-. Following incubation, the PBS was removed, and $1 \mathrm{ml}$ of siRNA complex was added to each flask. The media of each flask was brought to $1.5 \mathrm{ml}$ by adding $0.5 \mathrm{ml}$ OPTIMEM media and the cells were incubated at $37^{\circ} \mathrm{C}$ in the dark for 5 hours after which the media was replaced with full media.
Western Blots. MSC cell lysates were prepared by washing T25 plates with ice cold PBS three times to remove media followed by addition of $200 \mu$ lice cold RIPA buffer (10 $\mu \mathrm{l} / \mathrm{ml}$ PMSF solution, $10 \mu \mathrm{l} / \mathrm{ml}$ sodium orthovanadate, and $10 \mu \mathrm{l} / \mathrm{ml}$ protease inhibitor cocktail). Cells were lifted using a cell scraper, collected in Eppendorf tubes, and incubated on ice for $5 \mathrm{~min}$. Lysate was then clarified by centrifugation at $8000 \mathrm{rcf}$ at $4^{\circ} \mathrm{C}$ for $10 \mathrm{~min}$. The supernatant containing soluble protein was transferred to clean tubes and total protein concentration was determined by microBCA (Thermo Scientific). Western blots were performed using BioRad's Mini Protean Tetra Cell apparatus for the electrophoresis and transfer. Briefly, 10-20 $\mu \mathrm{g}$ of protein, mixed $1: 1$ with Laemmli Buffer were loaded into each well of $10 \%$ Tris- $\mathrm{HCl}$ gels with Tris running buffer for SDS-PAGE. Proteins were then transferred to a Polyvinyl difluoride membrane using the transfer apparatus according to the BioRad protocol. Following transfer, the membrane was incubated with 5\% BSA in TBST buffer overnight, washed with TBST and incubated with primary antibodies (rabbit antiIDO (12006S), rabbit anti-stat-1 (9172P), rabbit anti-pstat-1 (7649P), rabbit antiFOXO3 (2497P), rabbit anti- $\beta$-actin (4970S), Cell Signaling). Horseradish peroxidase conjugated anti-rabbit antibody (Cell Signaling, 7074S) was used as a secondary and protein bands were visualized following incubation with Amersham ECL Prime Detection Reagent (GE Healthcare) according to the manufacturer's instructions. Beta actin staining was performed to determine relative protein expressions and a Precision Plus Protein Dual Color Standard (Bio-Rad) was used to determine the molecular weight of the bands. Western blot images were analyzed and processed using ImageJ (NIH).

Budesonide Particle Formulation. PLGA particles were formulated using an oil-inwater single emulsion technique. $10 \mathrm{kDa}$ molecular weight $50: 50$ poly(lactic-coglycolic acid) with a carboxylic acid end group (inherent viscosity $0.15-0.25 \mathrm{dL} / \mathrm{g}$ ) was obtained from Lactel Absorbable polymers. $50 \mathrm{mg}$ of PLGA, $8 \mathrm{mg}$ of budesonide, and $10 \mu \mathrm{l}$ of DiI, were dissolved in $2 \mathrm{ml}$ of dichloromethane in a glass vial. After dissolving, PLGA:Drug solution was probe sonicated for 30 seconds to thoroughly mix the drug within the polymer. The solution was then added drop wise to $20 \mathrm{ml}$ of filtered $1 \%(\mathrm{w} / \mathrm{v})$ polyvinyl alcohol ( $80 \%$ hydrolyzed, Sigma) on ice while homogenizing at 33,000 rpm using a Tissue Master 125 homogenizer (Omni International). After 2 min homogenization, particles suspensions were gently stirred in a chemical hood for 4 hours to allow for evaporation of solvent. Suspensions were then centrifuged, and washed with distilled water 3 times before lyopholization and characterization. Blank particles were formed in parallel by omitting the addition of budesonide in the above procedure. The zeta-potential of particles was modified through adsorption of poly-l-lysine (PLL). Briefly, $6 \mathrm{mg} / \mathrm{ml}$ particle suspensions were prepared in $100 \mu \mathrm{g} / \mathrm{ml}$ PLL solution in distilled water and gently agitated for 2 hours. Particles were then frozen for subsequent use. The hydrodynamic diameter, polydispersity, and zeta potential of particles was measured in distilled water using a Malvern Zetaziser ZS90. Averages from three separate samples are reported. In addition, $40 \times$ fluorescence microscopy images were acquired to confirm the size and polydispersity of the particle suspensions.

Budesonide Particle Loading and Release Kinetics. High pressure liquid chromatography (HPLC) was used to determine the drug loading, encapsulation efficiency, and release kinetics of the budesonide microparticles. To determine drug loading, 2-3 mg of particles were weighed into Eppendorf tubes, quickly spun into a pellet, and swollen by addition of $500 \mu \mathrm{l}$ methanol. Samples were agitated at $37^{\circ} \mathrm{C}$ for 2 hours to allow for complete release of budesonide from the particles. Samples were centrifuged to pellet PLGA, and supernatant was collected, filtered, and analyzed by an Agilent 1100 series HPLC. An Agilent Zorbax Eclipse XDB-C18 column ( $4 \times$ $250 \mathrm{~mm}, 5 \mu \mathrm{m}$ ) was used with a mobile phase composed of $70: 30$ acetonitrile: $0.1 \%$ acetic acid, $25 \mu \mathrm{l}$ injection volume, and $1 \mathrm{ml} / \mathrm{min}$ flow rate. Budesonide was detected by peak absorbance at $248 \mathrm{~nm}$ and quantified by comparison to internal standard curves.

Drug loading was calculated as the dry mass of drug per mg of PLGA particles. Encapsulation efficiency was calculated as the ratio of encapsulated drug to total drug added to formulate particles. All samples were prepared in triplicate. Release kinetics were determined by suspending $2 \mathrm{mg}$ of particles in $200 \mu \mathrm{l}$ of PBS placed in a 2 inch section of 6-8,000 molecular weight cut off dialysis bag (Spectra Labs). Dialysis bags were submerged in $40 \mathrm{ml}$ of PBS to simulate infinite sync conditions and agitated at $37^{\circ} \mathrm{C}$. At each time point, $1 \mathrm{ml}$ of PBS was collected and replaced with fresh PBS. Samples were frozen until HPLC analysis using the method as described above. All samples were prepared in triplicate.

Particle Modification of MSCs. MSC particle modification was performed as previously described ${ }^{38}$. Briefly, MSCs were cultured to $80 \%$ confluence prior to particle modification. PLL coated budesonide or blank particles were thawed, briefly sonicated to break up particle clumps, and suspended in 1\% supplement StemPro culture media to make $10 \mathrm{ml}$ of $0.1 \mathrm{mg} / \mathrm{ml}$ particle media for each T75 flask. Flasks were modified at the end of the day and incubated with particle media overnight. Following particle modification, MSCs were washed three times with PBS, provided with fresh full supplement culture media and allowed to rest for 24 hours before subsequent experimentation. To confirm particle internalization, confocal microscopy was performed as previously described ${ }^{39}$.

PBMC Isolation from whole blood. Fresh whole blood from 3 donors was collected, after informed consent was obtained, from Research Blood Components for each experiment (Watertown, MA). Upon delivery, blood was diluted $1: 1$ with sterile 
PBS-/- supplemented with $2 \%$ fetal bovine serum, layered on top of Ficoll-Paque Premium (GE Healthcare), and centrifuged at $400 \mathrm{rcf}$ for $30 \mathrm{~min}$ with the brake off. The buffy coat was collected and washed with PBS:FBS solution. PBMCs were counted and either used immediately or frozen in freezing media (RPMI supplemented with 10\% DMSO, 40\% FBS, 1\% Penicillin/Streptomycin, 1\% L-Glut).

MSC:PBMC Co-cultures. The ratio of MSCs to PBMCs was established by adding 260,000 PBMCs and 65,000,32,500, or 16,250 MSCs to each well of a 24-well plate. MSCs were particle modified and allowed to rest a day before the co-culture experiments. Unmodified, blank-particle modified, or bud-particle modified MSCs were plated in 24 well plates and allowed to adhere. PBMCs labeled with a CellTrace CFSE Cell Proliferation Kit (Invitrogen) according to the manufacturer's instructions. 260,000 PBMCs were then added to each well. To stimulate PBMCs proliferation, 260,000 CD3 +/CD28 + Dynabeads (StemCell Technologies) were also added to each well. Total culture volume of each well was standardized to $0.5 \mathrm{ml}$ of RPMI supplemented with $10 \%$ FBS, $1 \%$ (v/v) L-Glutamine $1 \%$

penicillin:streptomycin. PBMCs stimulated with Dynabeads but without MSCs were used as an activated control and un-stimulated PBMCs grown without MSCs were used as an un-activated control for each donor (Fig 7A,B). Co-cultures were maintained for 5 days after which the media from the wells (containing PBMCs) was collected and centrifuged to pellet the PBMCs. The conditioned media was then collected for subsequent analysis. PBMC pellets were re-suspended in PBS-/containing 1\% FBS and PBMC proliferation was assessed by flow cytometery. Unstimulated PBMC controls were used to set the threshold for PBMC activation for each donor. To inhibit IDO activity, the MSC:PBMC co-cultures were repeated with or without the addition $1 \mathrm{mM}$ of the enzymatic inhibitor 1-methyl-DL-tryptophan (1-MT, Sigma) prepared in RPMI media. Data was analyzed using Accuri's CFlow software. IFN- $\gamma$ content of co-culture supernatant was determined using an ELISA MAX Deluxe Human IFN- $\gamma$ kit (Biolegend) by comparing to internal standards according to manufacturer's instructions.

Statistical Analysis. All statistics were performed using Prism 6 (GraphPad). Twoway ANOVA's were performed on data sets with two independent variables (dose and time or MSC group and MSC:PBMC ratios) and one-way ANOVA was performed on data sets with a single independent variable (effect of budesonide conditioning on MSC IDO activity). Fisher's LSD test without correction for multiple comparisons was used when assessing effect of budesonide doses on MSC metabolic activity and viability as the test has a higher Type I error and would therefore highlight any potential negative impact of budesonide on MSCs that would require further evaluation. Bonferroni correction for multiple comparisons, which is a more conservative test than the Fisher's LSD method, was used for the MHC expression data as all comparisons were made with respect to the untreated control. For all other statistics, the mean of all groups were compared to all other groups and thus, Tukey correction for multiple comparisons was used to minimize Type I error.

1. Ankrum, J. \& Karp, J. Mesenchymal stem cell therapy: Two steps forward, one step back. Trends Mol Med 16, 203-209 (2010).

2. Bahr von, L. et al. Long-term complications, immunologic effects, and role of passage for outcome in mesenchymal stromal cell therapy. Biol. Blood Marrow Transplant. 18, 557-564 (2012)

3. Le Blanc, K. et al. Mesenchymal stem cells for treatment of steroid-resistant severe, acute graft-versus-host disease: a phase II study. Lancet 371, 1579-1586 (2008).

4. Mills, C. R. Osiris Therapeutics Announces Preliminary Results for PRochymal Phase III GvHD Trials. Osiris Press Release (2009)at < http://files.shareholder. com/downloads/OSIR/2468414599x0x317779/7677da46-286a-47c4-865d36c148119a1a/OSIR_News_2009_9_8_General.pdf $>$.

5. Galipeau, J. The mesenchymal stromal cells dilemma-does a negative phase III trial of random donor mesenchymal stromal cells in steroid-resistant graftversus-host disease represent a death knell or a bump in the road? Cytotherapy 15, 2-8 (2013).

6. Melief, S. M., Zwaginga, J. J., Fibbe, W. E. \& Roelofs, H. Adipose Tissue-Derived Multipotent Stromal Cells Have a Higher Immunomodulatory Capacity Than Their Bone Marrow-Derived Counterparts. Stem Cells Translational Medicine doi:10.5966/sctm.2012-0184 (2013).

7. François, M., Romieu-Mourez, R., Li, M. \& Galipeau, J. Human MSC suppression correlates with cytokine induction of indoleamine 2,3-dioxygenase and bystander M2 macrophage differentiation. Mol Ther 20, 187-195 (2012).

8. Zhukareva, V., Obrocka, M., Houle, J. D., Fischer, I. \& Neuhuber, B. Secretion profile of human bone marrow stromal cells: donor variability and response to inflammatory stimuli. Cytokine 50, 317-321 (2010).

9. Prockop, D. J. Two Negative Feedback Loops Place Mesenchymal Stem/Stromal Cells (MSCs) at the Center of Early Regulators of Inflammation. Stem Cell doi:10.1002/stem.1400 (2013).

10. Lee, R. H. et al. Intravenous hMSCs Improve Myocardial Infarction in Mice because Cells Embolized in Lung Are Activated to Secrete the Anti-inflammatory Protein TSG-6. Cell Stem Cell 5, 54-63 (2009).

11. Kidd, S. et al. Direct evidence of mesenchymal stem cell tropism for tumor and wounding microenvironments using in vivo bioluminescent imaging. Stem Cell 27, 2614-2623 (2009).
12. Strioga, M., Viswanathan, S., Darinskas, A., Slaby, O. \& Michalek, J. Same or not the same? Comparison of adipose tissue-derived versus bone marrow-derived mesenchymal stem and stromal cells. Stem Cells Dev 21, 2724-2752 (2012).

13. Ren, G. et al. Species Variation in the Mechanisms of Mesenchymal Stem CellMediated Immunosuppression. Stem Cell 27, 1954-1962 (2009).

14. Nauta, A. J. \& Fibbe, W. E. Immunomodulatory properties of mesenchymal stromal cells. Blood 110, 3499-3506 (2007).

15. Bubnoff, D. \& Bieber, T. The indoleamine 2,3-dioxygenase (IDO) pathway controls allergy. Allergy 67, 718-725 (2012).

16. Meisel, R. et al. Human but not murine multipotent mesenchymal stromal cells exhibit broad-spectrum antimicrobial effector function mediated by indoleamine 2,3-dioxygenase. Leukemia 25, 648-654 (2011).

17. Munn, D. H. \& Mellor, A. L. Indoleamine 2,3 dioxygenase and metabolic control of immune responses. Trends in Immunology 34, 137-143 (2013).

18. Munn, D. H. et al. Prevention of allogeneic fetal rejection by tryptophan catabolism. Science 281, 1191-1193 (1998).

19. Prendergast, G. C., Metz, R. \& Muller, A. J. Towards a genetic definition of cancerassociated inflammation: role of the IDO pathway. Am. J. Pathol. 176, 2082-2087 (2010).

20. Munn, D. H. et al. GCN2 kinase in T cells mediates proliferative arrest and anergy induction in response to indoleamine 2,3-dioxygenase. Immunity 22, 633-642 (2005).

21. Terness, P. et al. Inhibition of allogeneic T cell proliferation by indoleamine 2,3dioxygenase-expressing dendritic cells: mediation of suppression by tryptophan metabolites. J. Exp. Med. 196, 447-457 (2002).

22. Fallarino, F. et al. IDO mediates TLR9-driven protection from experimental autoimmune diabetes. J Immunol 183, 6303-6312 (2009).

23. Grohmann, U. et al. Reverse signaling through GITR ligand enables dexamethasone to activate IDO in allergy. Nat Med 13, 579-586 (2007).

24. Puccetti, P. \& Grohmann, U. IDO and regulatory T cells: a role for reverse signalling and non-canonical NF-kappaB activation. Nat Rev Immunol 7, 817-823 (2007).

25. Türck, J., Oberdörfer, C., Vogel, T., Mackenzie, C. R. \& Däubener, W. Enhancement of antimicrobial effects by glucocorticoids. Med. Microbiol. Immunol. 194, 47-53 (2005).

26. Kebriaei, P. et al. Adult human mesenchymal stem cells added to corticosteroid therapy for the treatment of acute graft-versus-host disease. Biol. Blood Marrow Transplant. 15, 804-811 (2009).

27. Fakih, El, R., Obi, G. A., Scholoff, A., Carrum, G. \& Kamble, R. T. Systemic effects of oral budesonide in hematopoietic transplant: implications of drug interaction with azoles. Bone Marrow Transplant 47, 1370-1371 (2012).

28. Webster, J. \& Cidlowski, J. Mechanisms of Glucocorticoid-receptor-mediated Repression of Gene Expression. Trends Endocrinol. Metab. 10, 396-402 (1999).

29. Hammer, S. et al. Glucocorticoids mediate differential anti-apoptotic effects in human fibroblasts and keratinocytes via sphingosine-1-phosphate formation. J. Cell. Biochem. 91, 840-851 (2004).

30. Spurzem, J. R., Sacco, O., Rossi, G. A., Beckmann, J. D. \& Rennard, S. I. Regulation of major histocompatibility complex class II gene expression on bovine bronchial epithelial cells. J. Lab. Clin. Med. 120, 94-102 (1992).

31. Le Blanc, K., Tammik, C., Rosendahl, K., Zetterberg, E. \& Ringdén, O. HLA expression and immunologic properties of differentiated and undifferentiated mesenchymal stem cells. Exp Hematol 31, 890-896 (2003).

32. Cheon, H. \& Stark, G. R. Unphosphorylated STAT1 prolongs the expression of interferon-induced immune regulatory genes. Proceedings of the National Academy of Sciences 106, 9373-9378 (2009).

33. Wu, Y. et al. Up-regulation and Sustained Activation of Statl Are Essential for Interferon- (IFN-)-induced Dual Oxidase 2 (Duox2) and Dual Oxidase A2 (DuoxA2) Expression in Human Pancreatic Cancer Cell Lines. J Biol Chem 286, 12245-12256 (2011).

34. Morrow, A. N., Schmeisser, H., Tsuno, T. \& Zoon, K. C. A Novel Role for IFNStimulated Gene Factor 3II in IFN- Signaling and Induction of Antiviral Activity in Human Cells. J Immunol 186, 1685-1693 (2011).

35. Lützner, N., Kalbacher, H., Krones-Herzig, A. \& Rösl, F. FOXO3 Is a Glucocorticoid Receptor Target and Regulates LKB1 and Its Own Expression Based on Cellular AMP Levels via a Positive Autoregulatory Loop. PLoS ONE 7, e42166 (2012).

36. Toogood, J. H. Side effects of inhaled corticosteroids. J. Allergy Clin. Immunol. 102, 705-713 (1998).

37. Buchman, A. L. Side Effects of Corticosteroid Therapy. Journal of Clinical Gastroenterology 33, 289 (2001).

38. Ankrum, J. et al. Engineering cells with intracellular agent-loaded microparticles to control cell phenotype. Nat Protoc 9, 233-245 (2014).

39. Sarkar, D., Ankrum, J., Teo, G. S. L., Carman, C. V. \& Karp, J. M. Cellular and extracellular programming of cell fate through engineered intracrine-, paracrine-, and endocrine-like mechanisms. Biomaterials 32, 3053-3061 (2011).

40. Xu, C. et al. Tracking Mesenchymal Stem Cells with Iron Oxide Nanoparticle Loaded Poly(lactide-co-glycolide) Microparticles. Nano Lett 12, 4131-4139 (2012).

41. Distelhorst, C. W. Recent insights into the mechanism of glucocorticosteroidinduced apoptosis. Cell Death Differ 9, 6-19 (2002). 
42. la Garza-Rodea, de, A, S. et al. Exploitation of herpesvirus immune evasion strategies to modify the immunogenicity of human mesenchymal stem cell transplants. PLoS ONE 6, e14493 (2011).

43. Soland, M. A. et al. Modulation of Human Mesenchymal Stem Cell Immunogenicity through Forced Expression of Human Cytomegalovirus US Proteins. PLoS ONE 7, e36163 (2012).

44. Dejean, A. S. et al. Transcription factor Foxo3 controls the magnitude of T cell immune responses by modulating the function of dendritic cells. Nature Immunology 10, 504-513 (2009).

45. Watkins, S. K. \& Hurwitz, A. A. FOXO3: A master switch for regulating tolerance and immunity in dendritic cells. oncoimmunology 1, 252-254 (2012).

46. Watkins, S. K. et al. FOXO3 programs tumor-associated DCs to become tolerogenic in human and murine prostate cancer. J. Clin. Invest. 121, 1361-1372 (2011).

47. Liu, H., Liu, L., Fletcher, B. S. \& Visner, G. A. Sleeping Beauty-based gene therapy with indoleamine 2,3-dioxygenase inhibits lung allograft fibrosis. The FASEB Journal 20, 2384-2386 (2006).

48. Ankrum, J., Ong, J.F. \& Karp, J. M. Mesenchymal stem cells: immune evasive, not immune privileged. Nat Biotechnol. 32(3), 252-260 (2006).

\section{Acknowledgments}

This work was supported by National Institutes of Health grant HL095722, a Department of Defense grant \# W81XWH-13-1-0305, and by a Movember Prostate Cancer Foundation
Challenge Award to J.M.K. J.A.A. was supported by the Hugh Hampton Young Memoria Fellowship. We would also like to thank Prof. Dan Anderson of MIT and Dr. Augustine Choi of Weill Cornell Medical College for their constructive feedback on the manuscript.

\section{Author contributions}

J.A.A. and J.M.K. designed research; J.A.A., R.G.D. and O.J.F. performed research; J.A.A., R.G.D., O.L., J.M.K. analyzed data; J.A.A., R.G.D. and J.M.K. wrote the manuscript.

\section{Additional information}

Competing financial interests: J.M.K. is a paid consultant for Sanofi and Stempeudics.

How to cite this article: Ankrum, J.A., Dastidar, R.G., Ong, J.F., Levy, O. \& Karp, J.M Performance-enhanced mesenchymal stem cells via intracellular delivery of steroids. Sci. Rep. 4, 4645; DOI:10.1038/srep04645 (2014)

This work is licensed under a Creative Commons Attribution-NonCommercialNoDerivs 3.0 Unported License. The images in this article are included in the article's Creative Commons license, unless indicated otherwise in the image credit; if the image is not included under the Creative Commons license, users will need to obtain permission from the license holder in order to reproduce the image. To view a copy of this license, visit http://creativecommons.org/licenses/by-nc-nd/3.0/ 